\title{
INTEGRAL AND BP COHOMOLOGIES OF EXTRASPECIAL $p$-GROUPS FOR ODD PRIMES
}

\author{
Nobuaki Yagita
}

\begin{abstract}
For each odd prime $p$, we see $B P^{\text {odd }}\left(B p_{+}^{1+4}\right)=0$ where $p_{+}^{1+4}$ is the extraspecial $p$ group of order $p^{5}$ and of exponent $p$.
\end{abstract}

\section{Introduction}

Let $G$ be a compact group and $B G$ its classifying space. All known examples of $B P^{*}(B G)$ are generated by even dimensional elements. Hence it is conjectured that $B P^{\text {odd }}(B G)=0$. In this paper we give new examples of $B P^{\text {odd }}(B G)=0$.

Throughout this paper, let $p$ be an odd prime number. Let $p_{+}^{1+2 n}$ be the extraspecial $p$-group of order $p^{1+2 n}$ and exponent $p$. (For $p=2$, the group $2_{+}^{1+2 n}$ is the $n$-th central product of the dihedral group $D_{8}$ of order 8 .) It is known that the Morava $K$-theory $K(k)^{\text {odd }}(B G)=0$ for $G=p_{+}^{1+2}, D_{8}$ in [T-Y2] and for $G=2_{+}^{1+4}$ in $[\mathrm{S}-\mathrm{Y}]$. By a theorem in $[\mathrm{R}-\mathrm{W}-\mathrm{Y}]$, we know $B P^{\text {odd }}(B G)=0$ for these cases.

For $m \geq 1$ or $m=\infty$, let us write the central product by

$$
G_{m}^{n}=\mathbf{Z} / p^{m} \times \mathbf{Z} / p p_{+}^{1+2 n}, \quad G_{\infty}^{n}=S^{1} \times \mathbf{Z} / p p_{+}^{1+2 n}
$$

so that $G_{1}^{n}=p_{+}^{1+2 n}$.

THEOREM 1.1. The homology $H^{*}\left(B G_{\infty}^{2} ; \mathbf{Z}\right)$ has no higher p-torsion, i.e., all elements are just p-torsion or torsion free.

TheOREM 1.2. For $m \geq 2$ or $m=\infty, K(k)^{\text {odd }}\left(B G_{m}^{2}\right)=0$ for all $k$, and hence $B P^{\text {odd }}\left(B G_{m}^{2}\right)=0$. For $m=1$, we have $B P^{\text {odd }}\left(B G_{1}^{2}\right)=0$.

In $\S 2$, we recall the Hochschild-Serre spectral sequence converging to $H^{*}\left(B G_{\infty}^{n} ; \mathbf{Z} / p\right)$, which was studied in [T-Y3]. In $\S 3$, we study the similar

2000 Mathematics Subject Classification. Primary 55P35, 57T25; Secondary 55R35, 57T05.

Key words and phrases. Chow ring, motivic cohomology, $B P$-theory, extraspecial $p$ group.

Received May 16, 2003; revised December 6, 2004. 
type spectral sequence but converging the integral cohomology $H^{*}\left(B G_{\infty}^{n}\right)$. D. Green also studied this spectral sequence $[\mathrm{G}]$. Transferred elements are studied in $\S 4$. The exponent of $H^{*}\left(B G_{m}^{n}\right)$ is also studied in this section. For $m \geq 2, K(k)^{\text {odd }}\left(B G_{m}^{2}\right)=0$ and $B P^{\text {odd }}\left(B G_{m}^{2}\right)=0$ are proved in $\S 5$ and $\S 6$ respectively. Here we show $K(k)^{*}\left(B G_{\infty}^{2}\right) \cong K(k)^{*} \otimes H\left(H^{*}\left(B G_{\infty}^{2} ; \mathbf{Z} / p\right) ; Q_{k}\right)$. The fact $B P^{\text {odd }}\left(B G_{1}^{2}\right)=0$ is showed in $\S 7$. Here we use facts that $K(1)^{\text {odd }}\left(B G_{1}^{2}\right)=0$ and that the Euler number of $K(1)^{*}\left(B G_{1}^{2}\right)$ is known, e.g., by Brunetti [B1]. In the last section, we study the relation $B P^{*}\left(B G_{m}^{2}\right)$ and the Chow ring $C H^{*}\left(B G_{m}^{2}\right)$.

Discussions with David Green, Björn Schuster, Maurizio Brunetti and Ergün Yalcin have been very helpfull. The author thanks them very much.

\section{The central product of $p_{+}^{1+2 n}$ and $S^{1}$}

Hereafter we assume that $p$ is an odd prime. The extraspecial $p$-group $G=p_{+}^{1+2 n}$ is the group such that its exponent is $p$, its center is $C \cong \mathbf{Z} / p$ and there is the extension

$$
0 \rightarrow C \stackrel{i}{\rightarrow} G \stackrel{\pi}{\rightarrow} V \rightarrow 0
$$

with $V=\oplus^{2 n} \mathbf{Z} / p$. Throughout this section, we assume $G=p_{+}^{1+2 n}$.

We can take generators $a_{1}, \ldots, a_{2 n}, c \in G$ such that $\pi\left(a_{1}\right), \ldots, \pi\left(a_{2 n}\right)$ (resp. $c$ ) make a base of $V($ resp. $C)$ such that

$$
\left[a_{2 i-1}, a_{2 i}\right]=c \text { and }\left[a_{2 i-1}, a_{j}\right]=1 \quad \text { if } j \neq 2 i .
$$

Take the cohomologies

$$
\begin{gathered}
H^{*}(B C ; \mathbf{Z} / p) \cong \mathbf{Z} / p[u] \otimes \Lambda(z), \quad \beta z=u, \\
H^{*}(B V ; \mathbf{Z} / p) \cong \mathbf{Z} / p\left[y_{1}, \ldots, y_{2 n}\right] \otimes \Lambda\left(x_{1}, \ldots, x_{2 n}\right)=S_{2 n} \otimes \Lambda_{2 n} \quad \beta x_{i}=y_{i},
\end{gathered}
$$

identifying the dual of $a_{i}$ (resp. $c$ ) with $x_{i}$ (resp. $z$ ). Then from (2.2) the central extension (2.1) is expressed by

$$
f=\sum_{i=1}^{n} x_{2 i-1} x_{2 i} \in H^{2}(B V ; \mathbf{Z} / p) .
$$

Hence $\pi^{*} f=0$ in $H^{2}(B G ; \mathbf{Z} / p)$. Consider the spectral sequence

$$
E_{2}^{* * *}=H^{*}\left(B V ; H^{*}(B C ; \mathbf{Z} / p)\right) \Rightarrow H^{*}(G ; \mathbf{Z} / p) \text {. }
$$

Then the first nonzero differential is $d_{2}(z)=f$ since $\pi^{*}(f)=0$. The next differential is

$$
d_{3}(u)=\beta f=z(1) \quad \text { with } z(1)=\sum y_{2 i-1} x_{2 i}-y_{2 i} x_{2 i-1} .
$$

However this spectral sequence is quite difficult to compute and we consider more easy case. 
Let $C_{m}=Z / p^{m}$ and $C_{\infty}=S^{1}$. Let us define the central product $G_{m}=$ $G \times{ }_{C} C_{m}$ so that its center is isomorphic to $C_{m}$.

Hereafter we always assume $p>n$ and let $\tilde{G}=G_{\infty}^{n}$.

We consider the spectral sequence

$$
\tilde{E}_{2}^{* * *}=H^{*}\left(B V ; H^{*}\left(B S^{1} ; \mathbf{Z} / p\right)\right)=S_{2 n} \otimes \Lambda_{2 n} \otimes \mathbf{Z} / p[u] \Rightarrow H^{*}(B \tilde{G} ; \mathbf{Z} / p) .
$$

Here $H^{*}\left(B S^{1}\right) \cong \mathbf{Z}[u]$. This spectral sequence $\tilde{E}_{r}^{*, *}$ is computed in [T-Y3] when $r<2 p(p-1)$ for general $n$ and all $r$ for $n=2$. We recall some necessary facts and explain briefly how to compute this spectral sequence.

Given a graded $\mathbf{Z} / p$-algebra $A$ and $z \in A^{\text {odd }}$, we define the homology $H(A, z)$ with the differential $d(a)=z a$ since $z^{2}=0$. The first nonzero differential in $\tilde{E}_{r}^{*, *}$ is $d_{3}(u)=\beta f=z(1)$ from the naturality for $G \subset \tilde{G}$. Hence we want to compute $H\left(S_{2 n} \otimes \Lambda_{2 n}, z(1)\right)$. For this, we use the following lemma taken from [T-Y3].

Lemma 2.1. Let $y, z \in A$, and $|z|=$ odd, $|y|=$ even. Let us consider the $\mathbf{Z} /$-algebra $A \otimes \Lambda(x)$ for $|x|=|z|-|y|$. Then we have an additive isomorphism

$$
H(A \otimes \Lambda(x), y x+z) \cong(H(A, z) / y)\{x\} \oplus \operatorname{Ker}(y \mid H(A, z))
$$

where $\operatorname{Ker}(y \mid H(A, z))$ is the $\mathbf{Z} / p$-submodule of $H(A, z)$ generated by the elements annihilated by the $y$-multiplication.

From this lemma, we have $H\left(S_{2 n} \otimes \Lambda_{1}, y_{2} x_{1}\right) \cong S_{2 n} /\left(y_{2}\right)\left\{x_{1}\right\}$. By induction we get

$$
E_{4}^{*, 2} \cong H\left(S_{2 n} \otimes \Lambda_{2 n}, z(1)\right) \cong Z / p\left\{x_{1} \cdots x_{2 n}\right\}=Z / p\left\{f^{n}\right\} \text { since } n<p .
$$

Since $\operatorname{Ker}(z) \cong \operatorname{Im}(z) \oplus H(A, z)$ for $z \in A^{\text {odd }}$, it is immediate that

Lemma 2.2. There is an isomorphism $(A / z) / H(A, z) \cong \operatorname{Im}(z) \subset A$. In particular, if $A$ is $w$-torsion free for $w \in A^{\text {even, }}$, then so is $(A / z) / H(A, z)$.

Apply this lemma with $A=S_{2 n} \otimes \Lambda_{2 n}, z=z(1), w=y_{1}$. Since $y_{1}$ is injective on $A$, so is on $A /(z+H(A, z))$. Since $f^{n}$ is $y_{i}$-torsion, there is no nonzero differential $d_{r}: \mathbf{Z} / p\left\{f^{n} u^{s}\right\} \rightarrow A / z$ for $r<2 p-1$.

Next nonzero differential is the Kudo's transgression

$$
d_{2 p-1}\left(z(1) \otimes u^{p-1}\right)=\beta P^{1} \beta f=w(1) \quad \text { with } w(1)=\sum y_{2 i-1}^{p} y_{2 i}-y_{2 i}^{p} y_{2 i-1} .
$$

By the above lemma with $w=w(1)$, we know $\operatorname{Ker}\left(d_{2 p-1} \mid \operatorname{Im}(z(1))\right)=0$. Moreover we need

LEMma 2.3. $d_{2 p-1}\left(f^{n} \otimes u^{p-1}\right)=n z(2) f^{n-1} \quad$ where $\quad z(2)=P^{1} z(1)=$ $\sum y_{2 i-1}^{p} x_{2 i}-y_{2 i}^{p} x_{2 i-1}$.

Proof. Since $\tilde{E}_{r}^{*, \text { odd }}=0$, the Bockstein operation maps from $\tilde{E}_{r}^{*, \text { even }}$ to $\tilde{E}_{r}^{*+1, \text { even }}$. The element $\beta\left(f^{n} u^{p-1}\right)=n \beta(f) f^{n-1} u^{p-1}$ goes to $n w(1) f^{n-1}$ by 
$d_{2 p-1}$. Since $\beta(z(2))=w(1)$, we know that $d_{2 p-1}\left(f^{n} u^{p-1}\right)=n z(2) f^{n-1}+a$ with $a \in \operatorname{Ker}(\beta)$. Since $x_{i} f^{n}=0$ and $x_{i} z(2) f^{n-1}=0$ in $S_{2 n} \otimes \Lambda_{2 n} /(z(1))$, we know also $x_{i} a=0$ and hence $\beta\left(x_{i} a\right)=y_{i} a=0$ but $\operatorname{Ker}\left(y_{i}\right)=Z / p\left\{f^{n}\right\}$. This means $a=0$.

Therefore we have the theorem and

TheOREM 2.4 ((3.7) in [T-Y3]). There is an isomorphism $u^{p}: \tilde{E}_{2 p}^{*, *} \rightarrow \tilde{E}_{2 p}^{*, *+2 p}$

$$
\tilde{E}_{2 p}^{*, 2 j} \cong\left\{\begin{array}{l}
S_{2 n} \otimes \Lambda_{2 n} /\left(z(1), w(1), z(2) f^{n-1}\right) \quad \text { if } j=0 \bmod (p) \\
\mathbf{Z} / p\left\{f^{n} \otimes u^{j}\right\} \quad 1 \leq j<p-1 \\
0 \quad j=p-1 .
\end{array}\right.
$$

By the transgression theorem, the next differential is $d_{2 p+1}\left(u^{p}\right)=z(2)$. Let $E=S_{2 n} \otimes \Lambda_{2 n} /(z(1), w(1))$. We want to know $H\left(E /\left(z(2) f^{n-1}\right), z(2)\right)$. First we note the additive isomorphism

$$
H\left(E /\left(z(2) f^{n-1}\right), z(2)\right) \cong H(E, z(2)) \oplus \mathbf{Z} / p\left\{f^{n-1}\right\} .
$$

By the similar but after some computations, we get

THEOREM 2.5 (Corollary 5.19 in [T-Y3]). The term $\tilde{E}_{2 p+2}^{*, 2 p} \cong H\left(E /\left(z(2) f^{n-1}\right)\right.$, $z(2))$ is generated by $f^{n-1} \otimes u^{p}$ as an $S_{2 n} \otimes \Lambda_{2 n}$-module and

$$
\begin{gathered}
\beta: H(E, z(2))^{\text {odd }} \cong H(E, z(2))^{\text {even }} /\left(\mathbf{Z} / p\left\{f^{n}\right\}\right) \\
H\left(E /\left(z(2) f^{n-1}\right), z(2)\right)^{\text {even }} \cong S_{2 n} /\left(y_{i}^{p} y_{j}-y_{j}^{p} y_{i} \mid i \neq j\right)\left\{f^{n-1}\right\} \oplus \mathbf{Z} / p\left\{f^{n}\right\} .
\end{gathered}
$$

Let $w(2)=P^{p} w(1)=\sum y_{2 i-1}^{p^{2}} y_{2 i}-y_{2 i-1} y_{2 i}^{p^{2}}$. It is known that $(w(1), w(2))$ is a regular sequence in $S_{2 n}$ [T-Y1]. By using this fact and Lemma 2.2, we see

THEOREM 2.6. (1) The multiplying by $w(2)$ is injective on

$$
E /(z(2))+\mathbf{Z} / p\left\{f^{n-1}\right\}+H(E, z(2)) \cong E /\left(z(2)+S_{2 n} \otimes \Lambda_{2 n}\left\{f^{n-1}\right\}\right)
$$

(2) The multiplying by $w(2)$ is zero on $H\left(E / z(2) f^{n-1}, z(2)\right)$.

By using the facts that $S_{2 n} /(w(1))$ is $w(2)$-free but $H\left(E /\left(z(2) f^{n-1}\right), z(2)\right)$ is not $w(2)$-free, we can prove (Section 6 in [T-Y3])

LEMmA 2.7. $\tilde{E}_{2 p+2}^{*, *} \cong \tilde{E}_{2 p(p-1)+1}^{*, *}$.

By the Kudo's transgression, $d_{2 p(p-1)+1}\left(z(2) u^{p(p-1)}\right)=w(2)$. However for general $n$, it is unknown yet $d_{2 p(p-1)+1}\left(f^{n-1} u^{p(p-1)}\right)$.

Let us use the notation such that

$$
a \doteq b \quad \text { means } \quad a=\lambda b \quad \text { for } 0 \neq \lambda \in \mathbf{Z} / p .
$$


For $n=2$, we know

$$
d_{2 p(p-1)+1}\left(f u^{p(p-1)}\right) \doteq w_{12}(2)^{\prime} \beta\left(x_{1} x_{2}\right)+w_{34}(2)^{\prime} \beta\left(x_{3} x_{4}\right)
$$

where $w_{i j}(2)^{\prime}=\left(y_{i}^{p^{2}} y_{j}-y_{j}^{p^{2}} y_{i}\right) /\left(y_{i}^{p} y_{j}-y_{j}^{p} y_{i}\right)$. Hereafter let us write by $w(2)^{\prime}$ the element $w_{12}(2)^{\prime}-w_{34}(2)^{\prime}$. When $n=2$, there is the another differential

$$
d_{2 p^{3}}\left(f^{2} u^{p^{2}-2}\right) \doteq z(3)=P^{p} z(2) .
$$

Thus we can compute $\tilde{E}_{\infty}^{* * *}$ for $n=2$.

THEOREM 2.8 ([T-Y3]). For the spectral sequence converging to $H^{*}\left(G_{\infty}^{2} ; \mathbf{Z} / p\right)$, we have the isomorphisms

$$
\begin{aligned}
& \tilde{E}_{\infty}^{*, 2 p j} \cong\left\{\begin{array}{l}
S_{4} \otimes \Lambda_{4} /\left(z(1), z(2), z(3), w(1), w(2), w(2)^{\prime} \beta\left(x_{1} x_{2}\right)\right), \quad j=0 \bmod (p) \\
H(E /(z(2) f), z(2)) \quad 0<j<p-1 \bmod (p) \\
\mathbf{Z} / p\left\{f^{2}\right\} \quad j=p-1 \bmod (p),
\end{array}\right. \\
& \tilde{E}_{\infty}^{*, 2 j} \cong\left\{\begin{array}{l}
\mathbf{Z} / p\left\{f^{2}\right\} \quad 0<j<p-1 \bmod (p) \text { and } j \neq p^{j}-2 \\
0 j=p-1 \bmod (p) \text { or } j=p^{2}-2 .
\end{array}\right.
\end{aligned}
$$

Given $H^{*}(B \tilde{G} ; \mathbf{Z} / p)($ or $H(B \tilde{G}))$, to compute $H^{*}\left(B G_{m}^{n} ; \mathbf{Z} / p\right)$ (or $\left.H^{*}\left(B G_{m}^{n}\right)\right)$ we use the following fibration induced from (2.1)

$$
S^{1}=\tilde{G} / G_{m}^{n} \rightarrow B G_{m}^{n} \rightarrow B \tilde{G} .
$$

The induced spectral sequence is

$$
E_{2}^{*, *}=H^{*}\left(B \tilde{G} ; H^{*}\left(S^{1} ; \mathbf{Z} / p\right)\right) \cong H^{*}(B \tilde{G} ; \mathbf{Z} / p) \otimes \Lambda(z) \Rightarrow H^{*}\left(B G_{m}^{n} ; \mathbf{Z} / p\right) .
$$

Let us write $d_{2} z=f^{\prime}$. When $m=1$ this $f^{\prime}=f$ but when $n>1, f^{\prime}=0$ (see Proposition 3.17 in [Y2]).

Lemma 2.9. As $S_{2 n}$-modules, $H^{*}\left(B G_{m}^{n} ; \mathbf{Z} / p\right)$ is isomorphic to

$$
\left\{\begin{array}{l}
\left(\operatorname{Ker}(f) \mid H^{*}(B \tilde{G} ; \mathbf{Z} / p)\{z\} \oplus H^{*}(B \tilde{G} ; \mathbf{Z} / p) /(f) \text { if } m=1\right. \\
H^{*}(B \tilde{G} ; \mathbf{Z} / p) \otimes \Lambda(z) \quad \text { if } m \geq 2 .
\end{array}\right.
$$

\section{Integral cohomology}

We consider the integral coefficient spectral sequence

$$
I E_{2}^{*, *}=H^{*}\left(B V ; H^{*}\left(B S^{1}\right)\right) \Rightarrow H^{*}(B \tilde{G}) .
$$

This spectral sequence is also studied in $[\mathrm{G}]$ by Green. First we note that $H^{*}(B V) \cong \operatorname{Im}(\beta) \subset H^{*}(B V ; \mathbf{Z} / p)$ since the cohomology $H\left(H^{*}(B V ; \mathbf{Z} / p), \beta\right) \cong$ $\mathbf{Z} / p\{1\}$. The cohomology $H\left(H^{*}(B V) ; z(1)\right)$ is given by $\mathrm{D}$. Green.

Lemma $3.1([\mathrm{G}]) . \quad H\left(H^{*}(B V), z(1)\right) \cong \mathbf{Z}\{p\} \oplus \mathbf{Z} / p\left\{z(1) f, \ldots, z(1) f^{n-1}\right\}$. 
Proof. Let $V^{\prime} \oplus(\mathbf{Z} / p)^{2} \cong V$. By induction we assume that

$$
H^{+}\left(H^{*}\left(B V^{\prime}\right), z(1)\right) \cong \mathbf{Z} / p\left\{z(1), z(1) f, \ldots, z(1) f^{n-2}\right\} .
$$

Considering the spectral sequence

$$
E_{2}^{*, *}=H\left(H^{*}\left(B(\mathbf{Z} / p)^{2}\right) ; H^{*}\left(B V^{\prime}\right)\right) \Rightarrow H^{*}(B V)
$$

we can write $\operatorname{gr} H^{*}(B V) \cong A \oplus B \oplus \mathbf{Z}\{1\}$ where $A=E_{2}^{*,+} \cong H^{*}\left(B V^{\prime}\right)^{+} \otimes$ $\mathbf{Z} / p\left[y_{1}, y_{2}\right] \otimes \Lambda\left(x_{1}, x_{2}\right)$ and $B=E_{2}^{+, 0} \cong\left(\mathbf{Z} / p\left[y_{1}, y_{2}\right] \otimes \Lambda(\beta)\right)^{+}$with $\beta=\beta\left(x_{1} x_{2}\right)$.

From Lemma 2.1, we have

$$
H(A, z(1)) \cong H\left(H^{*}\left(B V^{\prime}\right)^{+}, z(1)\right)\left\{x_{1} x_{2}\right\}
$$

and $H(B, z(1)) \cong \mathbf{Z} / p\{\beta\}$ since $1 \notin B$ and $z(1) \mid B=\beta$. Thus we get

$$
H\left(\operatorname{gr} H^{*}(B V)^{+}, z(1)\right) \cong \mathbf{Z} / p\left\{\beta, z(1) x_{1} x_{2}, \ldots, z(1) f^{n-2} x_{1} x_{2}\right\} .
$$

Since $z(1) f^{i}$ is really cycle for the differential $z(1)$, and we have the lemma from

$$
H\left(H^{*}(B V)^{+}, z(1)\right) \cong H\left(H^{*}(B V), z(1)\right) \oplus \mathbf{Z} / p\{z(1)\} .
$$

COROLlaRY 3.2. The term $I E_{4}^{*, 2 i}$ is isomorphic to

$$
\left\{\begin{array}{l}
\mathbf{Z}\{1\} \oplus \beta H^{*}(B V ; \mathbf{Z} / p) /\left(z(1) \beta H^{*}(B V ; \mathbf{Z} / p)\right) \quad 2 i=0 \\
\mathbf{Z}\{p\} \oplus \mathbf{Z} / p\left\{z(1) f, \ldots, z(1) f^{n-1}\right\} \quad 0<2 i<2(p-1) \\
\mathbf{Z}\{p\} \oplus z(1) \beta H^{*}(B V ; \mathbf{Z} / p) \oplus \mathbf{Z} / p\left\{z(1) f, \ldots, z(1) f^{n-1}\right\} \quad 2 i=2 p-2 .
\end{array}\right.
$$

We use the following notations. For an element $a \in E_{\infty}^{*, *}$ converging to $H^{*}(X)$ (or $H^{*}(X ; Z / p)$ ), let us write by $\{a\}$ one of the correspondences elements in $H^{*}(X)$ (or $H^{*}(X ; \mathbf{Z} / p)$ ). For an element $x \in H^{*}(X)$, let $[x] \in E_{\infty}^{* * *}$ be the corresponding nonzero element in the spectral sequence. Therefore $[\{a\}]=a$ for $a \neq 0$ but $x \equiv\{[x]\}$ modulo $\left\{E^{*+1, *}\right\}$.

Let $r: H^{*}(X) \rightarrow H^{*}(X ; \mathbf{Z} / p)$ be the reduction map.

Lemma 3.3. Let $1 \leq s \leq n$. Then $d_{2 i+1}\left(p^{i-1} u^{s}\right) \doteq z(1) f^{i-1} u^{s-i}$ for all $i \leq s$, and $p^{s} u^{s}$ generates $I E_{2 s+2}^{0,2 s} \cong I E_{\infty}^{0,2 s}$. Moreover $r\left(\left\{p^{s} u^{s}\right\}\right)=f^{s}$.

Proof. By the naturality for the reduction map $r, d_{3}(u)=z(1)$ also in $I E_{3}^{*, *}$. Hence $p u \in E_{4}^{0,2}$ generates $E_{\infty}^{0,2}$ and $r(\{p u\}) \neq 0$. But it is easily seen that $\operatorname{Ker}(\beta) / \operatorname{Im}(\beta) \cap E_{\infty}^{2,0} \cong \mathbf{Z} / p\{f\}$. Thus we can take $r(\{p u\}) \doteq f$. For $s \leq n$, we have

$$
r\left(\left\{p^{s} u^{s}\right\}\right)=r(\{p u\})^{s} \doteq\{f\}^{s}=f^{s} .
$$

This means $p^{s} u^{s}$ generates $E_{\infty}^{0,2 s}$, and by dimensional reason, we have $d_{2 i+1}\left(p^{i-1} u^{s}\right) \doteq z(1) f^{i-1} u^{s-i}$ for all $i<s$.

Similarly, we have 
COHOMOLOGY OF EXTRASPECIAL $p$-GROUPS FOR ODD PRIMES

Lemma 3.4. Let $1 \leq i \leq n$ and $n \leq s \leq p-1$. Then $d_{2 i+1}\left(p^{i-1} u^{s}\right) \doteq$ $z(1) f^{i-1} u^{s-i}$.

For the proof of this lemma, we prepare the following lemma.

Lemma 3.5. Let $A$ be a graded algebra acting the Bockstein $\beta$ with $H(A, \beta)=0$. Let $z \in A^{\text {odd }}$ with $\beta z=0$ and write $H(A, z)=H$ and $H(\beta A, z)=$ IH. Then

$$
H(A /(z+H), \beta) \subset z^{-1} I H, \quad \operatorname{Im}(\beta)(A /(z+H)) \cong \beta A /(z \beta A+I H)
$$

identifying $z^{-1} I H$ as the submodule of $A /(z+H) \cong A / \operatorname{Ker}(z)$.

Proof. We note that

$\operatorname{Ker}(\beta)|(A /(z+H)) \cong \operatorname{Ker}(\beta)|(A / \operatorname{Ker}(z)) \stackrel{\times z}{\cong} \operatorname{Im}(z) \cap \operatorname{Ker}(\beta) \subset A$

On the other hand,

$$
\operatorname{Im}(\beta)(A /(z+H)) \stackrel{\times z}{\cong} \operatorname{Im}(\beta)(\operatorname{Im}(z)) \cong \operatorname{Im}(z)(\operatorname{Im}(\beta)) \cong \operatorname{Im}(z)(\operatorname{Ker}(\beta))
$$

since $\beta(z a)=z \beta(a)$ and $H(A, \beta)=0$. Thus we get

$$
\begin{aligned}
H(A /(z+H), \beta) & \stackrel{\times z}{\cong}(\operatorname{Im} z \cap \operatorname{Ker}(\beta)) / \operatorname{Im}(z)(\operatorname{Ker}(\beta)) \\
& \subset(\operatorname{Ker}(z) \cap \operatorname{Ker}(\beta)) / \operatorname{Im}(z)(\operatorname{Ker}(\beta))=H(\operatorname{Ker}(\beta), z) .
\end{aligned}
$$

Moreover we have

$$
\beta A /(z \beta A+I H) \cong \beta A / \operatorname{ker}(z) \stackrel{\times z}{\cong} \operatorname{Im}(z)(\operatorname{Im} \beta) .
$$

Let us write $A=E_{2}^{*, 0} \cong S_{2 n} \otimes \Lambda_{2 n}, B=E_{4}^{*, 0} \cong A / z(1)$, and $I A=I E_{2}^{+, 0} \cong \beta A$, $I B=I E_{4}^{+, 0} \cong I A /(z(1) I A)$. From the above lemma. We have

COROllary 3.6. $H\left(B^{+}, \beta\right) \cong Z / p\left\{f, \ldots, f^{n}\right\}$ and $I B / I H \cong \beta B$ where $I H \cong$ $\mathbf{Z} / p\left\{z(1) f, \ldots, z(1) f^{n-1}\right\}$.

Proof. Here $H\left(A^{+}, \beta\right)=H=0$ and hence

$$
H\left(B^{+}, \beta\right)=H\left(A^{+} / z(1), \beta\right)=H\left(A^{+} /(z(1)+H), \beta\right) \subset z(1)^{-1} I H,
$$

where $I H \cong \mathbf{Z} / p\left\{z(1) f, \ldots, z(1) f^{n-1}\right\}$ is still given in Lemma 3.1. Since $\beta f=$ $z(1)=0$ in $B, f^{i}$ are in $\operatorname{Ker}(\beta)$.

Let us write $\Delta=H\left(B^{+}, \beta\right) \cong Z / p\left\{f, \ldots, f^{n}\right\}$.

Proof of Lemma 3.4. From Theorem 2.4, we know for $*<2(p-1)$, $\operatorname{Ker} \beta\left(H^{*}(B \tilde{G} ; \mathbf{Z} / p)^{+}\right) \cong \beta B \oplus \Delta \oplus \mathbf{Z} / p\left\{f^{n} u, \ldots, f^{n} u^{p-2}\right\}$.

This module is also isomorphic to $H^{*}(B G) / p$. For each $i \leq p-1, p^{s} u^{i}$ are in 
$\tilde{E}_{\infty}^{0, *}$ for sufficient large $s$. Hence there is $s^{\prime}$ such that $r\left\{p^{s^{\prime}} u^{i}\right\} \doteq\left\{f^{n} u^{i-n}\right\}$, when $n \leq i \leq p-1$. Moreover each element of form $z(1) f^{k} u^{i}$ must be killed in the spectral sequence $I E_{\infty}^{*, *}$. By dimensional reason, we have the lemma. q.e.d.

Next consider differentials for elements in $I E_{4}^{*, 2(p-1)}$. The fact that $I E_{4}^{*, 2(p-1)} \cong \operatorname{Ker}(z(1)) \cap I A$ is $y_{i}$-torsion free implies that there does not exist differential such that $d_{r}(x) \neq 0 \in E_{r}^{*, 2(p-1)}$ for $4 \leq r \leq 2 p-1$ since $z(1) f^{i} u^{s}$ is $y_{j^{-}}$ torsion. Similarly since $A /(z+H) \cong B / H$ is $y_{j}$-torsion free, and so is $I B / I H \cong$ $\beta B / H$. Hence each element $z(1) f^{i} u^{s}$ does not go by differential into a nonzero element in $I B / I H$.

For the element $w(1) \in I E_{\infty}^{*, 0}$, since $r(w(1))=0 \in \tilde{E}_{\infty}^{*, 0}$, we have $w(1)=$ $\lambda p\left\{p^{s^{\prime}} u^{p+1}\right\}$ in $H^{*}(B \tilde{G})$ where note $\left|z(1) f^{i} u^{s}\right|=o d d$. But $w(1)$ is $p$-torsion also in $H^{*}(B \tilde{G})$ and $\left\{p^{s^{\prime}} u^{p+1}\right\}$ is torsion free and $\lambda=0$. Therefore there is an element $z$ with $d_{r}(z)=w(1)$ in $I E_{r}^{*, *}$. By dimensional reason or by naturality, we have

$$
d_{2 p-1}\left(z(1) u^{p-1}\right)=w(1)
$$

Similarly we get

$$
d_{2 p-1}\left(z(1) f^{i-1} u^{p-1}\right)=\beta\left(z(2) f^{i-1}\right)=w(1) f^{i-1}-(i-1) z(2) z(1) f^{i-2} .
$$

Recall that $E=S_{2 n} \otimes \Lambda_{2 n} /(z(1), w(1))$. Let us write $I E=I E_{2 p+1}^{+, 0}=$ $I B /(w(1) I B, \Gamma)$ where

$$
\Gamma=\mathbf{Z} / p\left\{\beta(z(2) f), \ldots, \beta\left(z(2) f^{n-1}\right)\right\} .
$$

LEMMA 3.7. $I E_{2 p+1}^{0,+} \cong I E \subset E /\left(z(2) f^{n-1}\right) \cong \tilde{E}_{2 p+1}^{0, *}$.

Proof. Let $x \in I B$ and $x=0 \in E$. Then $x=\beta\left(x^{\prime}\right)=w(1) a$ in $B$. Hence $w(1) \beta a=0$. Here $\operatorname{Ker}(w(1)) \cong \operatorname{Im} z(1) \oplus \mathbf{Z} / p\left\{f^{n}\right\}$. So $\beta a=\lambda f^{n}$ but it does not hold $\lambda \neq 0$ in $B$, indeed, $f^{n} \notin \beta B$. Thus $\beta a=0$. This means $a=\beta a^{\prime}+$ $\sum \lambda_{i} f^{i}$. Therefore

$$
\begin{aligned}
w(1) a & =w(1) \beta a^{\prime}+\sum w(1) \lambda_{i} f^{i} \\
& =w(1) \beta a^{\prime}+\sum \lambda_{i}\left(w(1) f^{i}-i z(2) z(1) f^{i-1}\right) \text { in } B
\end{aligned}
$$

since $z(1)=0 \in B$. Thus we see that $x \in(w(1) I B, \Gamma)$ and $x=0$ in $I E$.

LEMMA 3.8. $H\left(E^{+} / z(2), \beta\right) \cong \Delta$.

Proof. Let $\quad x \in \operatorname{Ker}(\beta \mid E / z(2))$. Since $\quad E /(z(2))=B /(w(1), z(2))$, this means

$$
\beta x=z(2) a+w(1) b \text { in } B .
$$

Take more $\beta$, and we get 


$$
0=\beta^{2} x=w(1) a-z(2) \beta a+w(1) \beta b .
$$

Multiply by $z(2)$, we have $z(2) w(1)(a+\beta b)=0$. Here we note that $\operatorname{Ker}(w(1))$ in $B$ is isomorphic to $\operatorname{Ker}(z(1)) \cong \operatorname{Im} z(1)+Z / p\left\{f^{n}\right\}$ in $A$. This fact is shown from that the Kudo's transgression $d_{2 p-1}: \operatorname{Im} z(1) \rightarrow B$ via. $z(1) \mapsto w(1)$ is injective. Hence $w(1) x=0$ in $B$ means that $z(1) x=0$ in $A$. By dimensional reason $|z(2)|>\left|f^{n}\right|$, we have $z(2)(a+\beta b)=0$. Thus

$$
\beta x=z(2)(-\beta b)+w(1) b=\beta(z(2) b) .
$$

Hence $\beta(x-z(2) b)=0$ in $B$. Since $H(B, \beta)=\Delta$, we have

$$
x-z(2) b \in \operatorname{Im} \beta+\Delta \text { in } B .
$$

Thus $x \in \operatorname{Im} \beta+\Delta$ in $E / z(2)$.

From Theorem 2.5, Lemma 2.7 and the above lemma, we see;

COROLlary 3.9. When $*<2 p^{2}-2 p$, each element of $H^{*}(B \tilde{G})$ is torsion free or just p-torsion.

From the above corollary, the map $r: I E_{\infty}^{m, 0} \rightarrow \tilde{E}_{\infty}^{m, 0}$ is injective for $0<$ $m<2 p^{2}-2 p$. In particular elements $\beta(z(2) x) \in \tilde{E}_{2 p-1}^{*, 0}, * \leq 2(p+n)<4 p-1$ must be target $d_{r}(z)$ for some $z \in \tilde{E}_{r}^{* * *}$ by arguments before Lemma 3.7. By the naturality, we see

$$
d_{2 p+1}\left(\beta(x) u^{p}\right)=\beta(x z(2)) \text { in } I E_{2 p+1}^{*, *}
$$

from the fact $d_{2 p+1}\left(x u^{p}\right)=x z(2)$ in $\tilde{E}_{r}^{* * *}$. For the cases $|\beta(x)| \geq 4 p-1$, we can write $x=\sum x^{\prime} \beta\left(x^{\prime \prime}\right)$ with $\left|x^{\prime}\right|<2 p$ and we also have $d_{2 p+1}\left(u^{p} \beta(x)\right)=z(2) \beta(x)$.

Let $F=\tilde{E}_{2 p+2}^{*, 0} \cong E /(z(2))$ and $I F=I E /(\beta(z(2) E))$.

LEMma 3.10. IF $\subset F$ and $\beta H(E, z(2)) \cong I H(I E, z(2))$ where $\operatorname{IH}(\operatorname{IE}, z(2))=$ $\{\beta(x) \in I E \mid \beta(z(2) x)=0\} /(\beta(z(2) E))$.

Proof. Let $\beta(x) \in I E$ and $\beta(x)=0 \in F$. From the proof of Lemma 3.7, we can see that $\beta(x)=z(2) \beta b-w(1) b=\beta(z(2) b) \in E$. So $x=0 \in I F$.

From the above corollary and lemma, we show that all nonzero elements in $I E_{r}^{*,+}$ $* \neq 0 \bmod (p)$ must be killed.

Lemma 3.11. When $s \leq n$, we get

$$
\begin{cases}d_{2 i+1}\left(p^{i-1} u^{s+p}\right) \doteq z(1) f^{i-1} u^{s+p-i} & \text { if } s \geq i \\ d_{2 s+1}\left(p^{s} u^{s+p}\right)=0 & \\ d_{2 i+3}\left(p^{i-1} u^{s+p}\right) \doteq z(1) f^{i} u^{s+p-i-1} & n \geq i>s+1\end{cases}
$$


COROllary 3.12 .

$$
\begin{aligned}
I E_{2 p(p-1)+1}^{*, 2 j} \cong\left\{\begin{array}{l}
I F \cong \mathbf{Z}\{1\} \oplus \beta E /(\beta(z(2) E)) \quad j=0 \\
\mathbf{Z}\left\{p^{j} u^{j}\right\} \quad 0<2 j<2 n \\
\mathbf{Z}\left\{p^{n} u^{j}\right\} \quad 2 n \leq 2 j \leq 2 p-2
\end{array}\right. \\
I E_{2 p(p-1)+1}^{*, 2 p+2 j} \cong\left\{\begin{array}{l}
\beta H(E, z(2)) \oplus \mathbf{Z}\left\{p^{n-1} u^{p}\right\} \quad j=0 \\
\mathbf{Z}\left\{p^{n-1} u^{p+j}\right\} \quad 0<2 j<2 n \\
\mathbf{Z}\left\{p^{n} u^{p+j}\right\} \quad 2 n \leq 2 j \leq 2 p-2 .
\end{array}\right.
\end{aligned}
$$

Now we consider the case $n=2$. Recall $w(2) /\left(y_{1}^{p} y_{2}-y_{1} y_{2}^{p}\right)=w_{12}(2)^{\prime}-$ $w_{34}(2)^{\prime}$ and write it by $w(2)^{\prime}$ so that $d_{2 p(p-1)}\left(f u^{p(p-1)}\right) \doteq w(2)^{\prime} \beta\left(x_{1} x_{2}\right) \in \tilde{E}_{r}^{*, 0}$. Hence $w(2)^{\prime} \beta\left(x_{1} x_{2}\right)=p a$ in $H^{*}(\tilde{G} ; \mathbf{Z})$. But nonzero elements in $I E_{2 p(p-1)+1}^{*, s}$ for $0<s<2 p^{2}$ are even dimensional from Cor. 3.12 and Theorem 2.5. Hence $w(2)^{\prime} \beta\left(x_{1} x_{2}\right)=0$ also in $H^{*}(\tilde{\boldsymbol{G}} ; \mathbf{Z})$. By dimensional reason we have

$$
d_{2(p-1) p+3}\left(p u^{p(p-1)+1}\right) \doteq w(2)^{\prime} \beta\left(x_{1} x_{2}\right) \quad \text { in } I E_{r}^{*, 0} .
$$

Define

$$
\begin{gathered}
G=E_{\infty}^{*, 0} \cong F /\left(w(2), w(2)^{\prime} \beta\left(x_{1} x_{2}\right), z(3)\right) \\
\text { and } I G=I F /\left(w(2)\{1, I F\}, w(2)^{\prime} \beta\left(x_{1} x_{2}\right) I F\right) .
\end{gathered}
$$

LEMma 3.13. When $n=2, I G=\beta G$ and $I G \cong I E_{\infty}^{+, 0}$. Moreover $H(G, \beta) \cong$ $\Delta \oplus \mathbf{Z} / p\left\{w(2)^{\prime} x_{1} x_{2}\right\}$.

Proof. Let $x=0 \in G$ and $x=\beta x^{\prime} \in F$. Then in $F$,

$$
\beta\left(x^{\prime}\right)=w(2) a+w(2)^{\prime} \beta\left(x_{1} x_{2}\right) c+z(3) d \quad \text { for } c \in H(E ; Z(2)), d \in \mathbf{Z} / p .
$$

By dimensional reason, we see $d=0$. First consider the case $|x|=$ even. Applying $\beta$, we see

$$
w(2) \beta a+w(2)^{\prime} \beta\left(x_{1} x_{2}\right) \beta(c)=0 .
$$

Here $|c|=o d d \quad$ and $\quad c=0$ otherwise $w(2)^{\prime} \beta\left(x_{1} x_{2}\right) \beta(c) \neq 0 \bmod (w(2))$ from Theorem 2.5 and Theorem 2.6 (2). Thus $w(2) \beta(a)=0$. Hence for this case, we can prove the lemma by the arguments similar to those of the proof of Lemma 3.9 .

Let $|x|=o d d$. Then $|c|=$ even and also from Theorem 2.5, $\beta c=0$ and $\beta\left(x_{1} x_{2}\right) c=\beta\left(x_{1} x_{2} c\right)$. Therefore we can prove the lemma similarly to the case $|x|=$ even.

Remark. The fact $H(G, \beta) \cong \Delta \oplus \mathbf{Z} / p\left\{w(2)^{\prime} x_{1} x_{2}\right\}$ is also proved in Section 4 below.

Thus we get the results for the case $n=2$.

THEOREM 3.14. When $n=2$ 
COHOMOLOGY OF EXTRASPECIAL $p$-GROUPS FOR ODD PRIMES

$$
I E_{\infty}^{*, 2 p j} \cong\left\{\begin{array}{l}
\mathbf{Z}\{1\} \oplus I G \quad \text { if } j=0 \bmod (p) \\
\mathbf{Z}\{p\} \oplus I H(E, z(2)) \quad 0<j<p-1 \bmod (p) \\
\mathbf{Z}\{p\} \quad j=p-1 \bmod (p),
\end{array}\right.
$$

For $j \neq 0 \bmod (p)$,

$$
I E_{\infty}^{*, 2 j} \cong\left\{\begin{array}{l}
\mathbf{Z}\{p\} \quad j=1 \bmod (p), j \neq p(p-1)+1 \bmod \left(p^{2}\right) \\
\mathbf{Z}\left\{p^{2}\right\} \quad 2 \leq j \leq p-1 \bmod (p) \text { or } j=p(p-1)+1 \bmod \left(p^{2}\right) .
\end{array}\right.
$$

Corollary 3.15. All elements in $H^{*}\left(B G_{\infty}^{2}\right)$ are just p-torsion or torsion free. by

COROLlary 3.16. The reduced map $r: H^{*}\left(B G_{\infty}^{2}\right) \rightarrow H^{*}\left(B G_{\infty}^{2} ; \mathbf{Z} / p\right)$ is given

$$
\left\{\begin{array}{l}
r\left\{p u^{s p}\right\} \doteq\left\{f^{2} u^{s p-2}\right\} \quad 1 \leq s \leq p-1 \\
r\left\{p u^{s p+1}\right\} \doteq\left\{f u^{s p}\right\} \quad 0 \leq s \leq p-2 \\
r\left\{p^{2} u^{p(p-1)+1}\right\} \doteq\left\{w(2)^{\prime} x_{1} x_{2}\right\} \\
r\left\{p^{2} u^{s p+j}\right\} \doteq\left\{f^{2} u^{s p+j-2}\right\} \quad 2 \leq j \leq p-1
\end{array}\right.
$$

Now we study the integral cohomology of the finite groups $G_{m}^{n}$. The integral version of the spectral sequence is

$$
I E_{2}^{*, *}=H^{*}(B \tilde{G}) \otimes \Lambda(z) \Rightarrow H^{*}\left(B G_{m}^{n}\right) .
$$

Here the differential is $d_{2}(z)=f^{\prime} \doteq\left\{p^{m} u\right\} \in H^{*}(B \tilde{G})$. This fact is proved by the naturality to the restriction maps

$$
S^{1} \rightarrow B \mathbf{Z} / p^{m} \rightarrow B S^{1}
$$

and by the isomorphism $H^{*}\left(B \mathbf{Z} / p^{m}\right) \cong \mathbf{Z}[u] /\left(p^{m} u\right)$. Similarly to the $\bmod p$ case, we have the isomorphism

$$
H^{*}\left(B G_{m}^{n}\right) \cong\left(\operatorname{Ker} f^{\prime} \mid H^{*}(B \tilde{G})\right) \oplus H^{*}(B \tilde{G}) /\left(f^{\prime}\right) .
$$

For the integral case, $d_{2}(z) \neq 0$ even if $m \geq 2$. Let $p^{m(i)} u^{i}$ generate $I E_{\infty}^{0, *}$. Since

$$
d_{2}\left\{p^{m(i-1)} u^{i-1} z\right\} \doteq\left\{p^{m(i-1)} u^{i-1}\right\}\left\{p^{m} u\right\}=\left\{p^{m(i-1)+m} u^{i}\right\},
$$

we have

$$
p^{m(i-1)+m-m(i)} \mid \exp \left(H^{*}\left(B G_{m}^{n}\right)\right)
$$

where $\exp \left(H^{*}\left(B G_{m}^{n}\right)\right)$ is the exponent of $H^{*}\left(B G_{m}^{n}\right)$.

Since each element of $H^{*}\left(B G_{\infty}^{2}\right)$ is just $p$-torsion or torsion free, and $m\left(p^{2}\right)=0$ and $m\left(p^{2}-1\right)=2$, we easily see that

Corollary 3.17. $\exp \left(H^{*}\left(B G_{m}^{2}\right)\right)=p^{m+2}$.

This fact is extended for all $n<p$ in Corollary 4.7 bellow. 


\section{Transfers}

In this section, we study about generators $p^{m(i)} u^{i} \in I E_{\infty}^{0,2 i}$. We can take $\left\{p^{s} u^{s}\right\}$ as a Chern class $c_{s}(\xi)$ where $\xi$ is a one dimensional representation with $\xi(x)=e^{2 \pi x i}$ for $x \in \mathbf{R} / \mathbf{Z} \cong S^{1}$ and $\xi\left(a_{j}\right)=1$. Moreover $\left\{p^{n} u^{n}\right\}$ is represented by transfer.

Let $A^{\text {odd }}$ be the maximal abelian subgroup of $\tilde{G}$ generated by

$$
A^{\text {odd }}=\left\langle a_{1}, a_{3}, \ldots, a_{2 n-1}\right\rangle \times S^{1}
$$

so that

$$
H^{*}\left(B A^{\text {odd }} ; \mathbf{Z} / p\right) \cong \mathbf{Z} / p\left[y_{1}, y_{3}, \ldots, y_{2 n-1}\right] \otimes \Lambda\left(x_{1}, x_{3}, \ldots, x_{2 n-1}\right) \otimes \mathbf{Z} / p[u] .
$$

Consider the transfer

$$
\operatorname{tr}(i)=\operatorname{Cor}_{A^{\text {odd }}}^{\tilde{G}}\left(u^{i}\right) \in H^{*}(B \tilde{G})=H^{*}\left(B G_{\infty}^{n}\right) .
$$

Since $\left[\tilde{\boldsymbol{G}} ; A^{\text {odd }}\right]=p^{n}$, we have $\operatorname{tr}(i) \mid S^{1}=p^{n} u^{i}$. Moreover $r(\operatorname{tr}(i))$ is $x_{\text {even }}$-torsion and $y_{\text {even }}$-torsion because by the Frobenius formula

$$
y_{\text {even }} \operatorname{tr}(i)=y_{\text {even }} \operatorname{Cor}\left(u^{i}\right)=\operatorname{Cor}\left(i_{\text {odd }}^{*}\left(y_{\text {even }}\right) u^{i}\right)=0
$$

where $i_{\text {odd }}: A^{\text {odd }} \rightarrow \tilde{G}$ is the inclusion and $i_{\text {odd }}^{*}\left(y_{\text {even }}\right)=0$.

Lemma 4.1. If $i \leq n(p-1)$, then $r(\operatorname{tr}(i)) \doteq\left\{f^{n} u^{i-n}\right\}$ or 0 .

Proof. The transfer $r(\operatorname{tr}(i))$ is $y_{\text {even }}$-torsion, and $x_{\text {even }}$-torsion in $H^{*}(B \tilde{G} ; \mathbf{Z} / p)$, and also in $E_{2 p^{2}+1}^{*, *}$ for $i<2 p^{2}$. Hence $r(\operatorname{tr}(i))$ is $w(2)=$ $\sum y_{2 i-1}^{p} y_{2 i}-y_{2 i}^{p} y_{2 i-1}$-torsion in $E_{2 p^{2}+1}^{* * *}$. From Theorem 2.6, there is no nonzero such torsion element in

$$
E_{2 p^{2}+1}^{*^{\prime}, *} /\left(H(E, z(2))+Z / p\left\{f^{n-1}\right\}\right) \quad \text { for } *^{\prime}<2 p(p-1)+1 .
$$

Also from Theorem 2.5 and Lemma 4.3 (2) below, the nonzero $y_{\text {even }}$-torsion elements of degree less than $2 n(p-1)+1$ is only the $f^{n}$ in $H(E, z(2))+$ $\mathbf{Z} / p\left\{f^{n-1}\right\}$.

From Corollary 3.12, we have (see also Lemma 4.6 bellow)

Corollary 4.2. If $n \leq i \leq(p-1) \bmod (p)$, then $r(\operatorname{tr}(i)) \doteq\left\{f^{n} u^{i-n}\right\}$.

Proof. Recall $\operatorname{tr}(i) \mid S^{1}=p^{n} u^{i}$. From Corollary 3.12, we know $\operatorname{tr}(i) \neq 0$ $\bmod (p)$ in $H^{*}(B \tilde{G})$. Hence $r(\operatorname{tr}(i)) \neq 0$. Thus we have the corollary from the above lemma.

Lemma 4.3. Given $k \geq 1$, let $a \in S=S_{l} /\left(y_{i}^{p^{k}} y_{j}-y_{i} y_{j}^{p^{k}} \mid 1 \leq i<j \leq l\right)$. Then we have

(1) if $y_{i} a=0$ for all $i$, then $a=0$. 
(2) if $a \in \operatorname{Ideal}\left(y_{1} \cdots y_{s}\right)$ and $y_{i} a=0$ for all $1 \leq i \leq s$, then $|a| \geq$ $s\left(p^{k}-1\right)+1$.

Proof. Replacing $y_{i} y_{j}^{p^{k}}$ by $y_{i}^{p^{k}} y_{j}$ for $i<j$, we can uniquely write an element $a \in S$ as

$$
a=\sum \lambda_{I} y_{I}=\sum \lambda y_{1}^{i_{1}} \cdots y_{l}^{i_{l}}
$$

where $I=\left(i_{1}, \ldots, i_{l}\right)=\left(0, \ldots, 0, i_{m(I)}, i_{m(I)+1}, \ldots, i_{l}\right)$ with $i_{m(I)} \neq 0$ and $0 \leq i_{s} \leq$ $p^{k}-1$ for all $m(I)+1 \leq s \leq l$.

For the proof of (1), let $\tilde{I}$ be the smallest $I$ for $\lambda_{I} \neq 0$ by the lexicographic order (i.e., $I>J$ if there is $s$ such that $i_{k}=j_{k}$ for all $k<s$ and $i_{s}>j_{s}$ ). Then $y_{m(\tilde{I})} a \neq 0$ because $y_{m(\tilde{I})} y_{I}>y_{m(\tilde{I})} y_{\tilde{I}}$ for $I>\tilde{I}$. This shows (1).

Suppose that $y_{i} a=0$ for $l-s \leq i \leq l$. Then $\tilde{i}_{l} \geq p^{k}-1$, otherwise $y_{l} y_{\tilde{I}}$ becomes the smallest in $y_{l} y_{I}$, and hence $y_{l} a \neq 0$. Since $a \in \operatorname{Ideal}\left(y_{l-s} \cdots y_{l}\right)$, we know $\tilde{i}_{l}=p^{k}-1$ if $s \geq 1$. Next applying $y_{l-1}$ on $a$ implies $\tilde{i}_{l-1}=p^{k}-1$ if $s \geq 2$. Continue this arguments, we know $\tilde{i}_{t}=p^{k}-1$ for $l-s \leq t \leq l$. This shows (2).

For a finite group $G$, an element $x \in H^{*}(B G ; \mathbf{Z} / p)$ is said to be essential if it restricts trivially to all proper subgroups of $G$. We consider essential elements for $G=G_{1}^{n}=p_{+}^{1+2 n}$. Similar arguments are also done by Minh ([Mi]).

Proposition 4.4. If $n<i<(p-1)$, then $\operatorname{tr}(i) \in H^{*}\left(B G_{1}^{n} ; \mathbf{Z} / p\right)$ is essential.

Proof. Any maximal subgroup $M$ of $G_{1}^{n}$ is isomorphic to $G_{1}^{n-1} \times \mathbf{Z} / p$. Let $\langle M, g\rangle=G_{1}^{n}$. Suppose that $A^{\text {odd }}=A \subset M$. Then by the double coset formula,

$$
\operatorname{tr}(i) \mid M=\sum_{k=0}^{p-1} \operatorname{Cor}_{g^{k} A g^{-k} \cap M}^{M}\left(g^{k *} u^{i}\right)=\operatorname{Cor}_{A}^{M}\left(\sum_{k} g^{k *} u^{i}\right) .
$$

Let $\quad H^{*}(M ; \mathbf{Z} / p) \cong H^{*}\left(B G_{1}^{n-1} ; \mathbf{Z} / p\right) \otimes \mathbf{Z} / p[y] \otimes \Lambda(x)$ so that $g^{*}(u)=u+y$. Then

$$
\sum_{k} g^{k *} u^{i}=\sum_{k}(y+k y)^{i}=\sum_{j=0}^{i}\left(\begin{array}{c}
i \\
j
\end{array}\right)\left(\sum k^{j}\right) u^{i} y^{i-j}=0 \bmod (p)
$$

since $\sum_{k=0}^{p-1} k^{j}=0 \bmod (p)$ for $j<p-1$.

Next suppose that $\langle A, M\rangle=G$. Let $\tilde{A}=A \cap M$. Then $\tilde{A} \cong(Z / p)^{s}$ for $s \leq n$. Since all maximal elementary abelian $p$-subgroup of $G_{1}^{n}$ have the rank $=$ $n+1$, there is a subgroup $A \cap M \subset B \subset M$ with $B \cong(Z / p)^{n+1}$. By the double coset formula, we also have

$$
\operatorname{tr}(i) \mid M=\operatorname{Cor}_{\tilde{A}}^{M}\left(u^{i}\right)=\operatorname{Cor}_{B}^{M} \operatorname{Cor}_{\tilde{A}}^{B}\left(u^{i}\right) .
$$

Since $B \cong \tilde{A} \times(\mathbf{Z} / p)^{n+1-s}$, we see $\operatorname{Cor}_{\tilde{A}}^{B}(-)=0$. 
Let $A^{\prime}=\left\langle A, G_{1}^{1}\right\rangle \subset G_{1}^{n}$. Then from Corollary 3.12 and Theorem 2.4, we see

$\left\{f^{n} u^{p-n}\right\} \doteq \operatorname{Cor}_{A^{\prime}}^{G_{1}^{n}}\left(u_{p}\right) \quad$ where $u_{p}=\left\{u^{p}\right\} \in H^{*}\left(B G_{1}^{1} ; \mathbf{Z} / p\right) \subset H^{*}\left(B A^{\prime} ; \mathbf{Z} / p\right)$.

Proposition 4.5. For $n \geq 2$, the element $\operatorname{Cor}_{A^{\prime}}^{G_{1}^{n}}\left(u_{p}\right) \in H^{*}\left(B G_{1}^{n} ; \mathbf{Z} / p\right)$ is essential.

Proof. Suppose that $A^{\prime} \subset M$. Then by the double coset formula

$$
\operatorname{Cor}_{A^{\prime}}^{G_{1}^{n}}\left(u_{p}\right) \mid M=\sum_{k=0}^{p-1} \operatorname{Cor}_{g^{k} A^{\prime} g^{-k} \cap M}^{M}\left(g^{k *} u_{p}\right)=\operatorname{Cor}_{A^{\prime}}^{M}\left(\sum_{k} g^{k *} u_{p}\right) .
$$

It is known that $u_{p} \mid\left\langle a_{1}, c\right\rangle=u^{p}-y_{1}^{p-1} u[\mathrm{~L}]$. Hence

$$
g^{*} u_{p} \mid A=(u+y)^{p}-y_{1}^{p-1}(u+y)=\left(u^{p}+y_{1}^{p-1} u\right)+\left(y^{p}-y_{1}^{p-1} y\right) .
$$

From this equation we can prove (for details, see $[\mathrm{L}]$ )

$$
g^{*} u_{p}=u_{p}+y^{p}-\chi y \quad \text { where } \chi=\operatorname{Cor}_{\left\langle a_{1}, c\right\rangle}^{G_{1}^{1}}\left(u^{p-1}\right)+y_{2}^{p-1} .
$$

Here we identify $\operatorname{Cor}_{\left\langle a_{1}, c\right\rangle}^{G_{1}^{1}}(-)=\operatorname{Cor}_{A}^{A^{\prime}}(-)$ since $\left\langle a_{1}, c\right\rangle \times(\mathbf{Z} / p)^{n-1} \cong A$ and $G_{1}^{1} \times(\mathbf{Z} / p)^{n-1} \cong A^{\prime}$. Thus we get $\sum_{k} g^{k *} u_{p}=0$ since $g^{*} \chi=\chi$.

Next suppose that $\left\langle A^{\prime}, M\right\rangle=G$. Let us write $\tilde{A}=A^{\prime} \cap M$. If $\operatorname{rank}_{p}(\tilde{A}) \leq n$, then we can take $B$ as the proof of Proposition 4.4. Similarly we get $\operatorname{Cor}_{\tilde{A}}^{B}(-)=0$ for the above case. Hence let $\tilde{A} \cong(\mathbf{Z} / p)^{n+1}$ and this implies $\tilde{A}=A$. Also by the double coset formula

$$
\operatorname{Cor}_{A^{\prime}}^{G_{1}^{n}}\left(u_{p}\right) \mid M=\operatorname{Cor}_{A}^{M}\left(u^{p}-y_{1}^{p-1} u\right)=\operatorname{Cor}_{A}^{M}\left(u^{p}\right)-y_{1}^{p-1} \operatorname{Cor}_{A}^{M}(u) \text {. }
$$

But the above formula is zero by the following reason. We take $\tilde{A}=A \subset$ $B \subset M$ such that $B \cong G_{1}^{1} \times(\mathbf{Z} / p)^{n-1}$. Here let us reorder $i$ of $a_{i}$ so that $B \supset$ $G_{1}^{1}=\left\langle c, a_{3}, a_{4}\right\rangle$. The restrictions

$$
\begin{gathered}
\operatorname{Cor}_{\left\langle a_{3}, c\right\rangle}^{G_{1}^{1}}\left(u^{p}\right) \mid\left\langle a_{3}^{\lambda} a_{4}, c\right\rangle=\operatorname{Cor}_{\langle c\rangle}^{\left\langle a_{3}^{\lambda} a_{4}, c\right\rangle}\left(u^{p}\right)=0 \quad \text { for } 0 \leq \lambda \leq p-1, \\
\operatorname{Cor}_{\left\langle a_{3}, c\right\rangle}^{G_{1}^{1}}\left(u^{p}\right) \mid\left\langle a_{3}, c\right\rangle=\sum_{k=0}^{p-1}\left(a_{4}\right)^{* k}\left(u^{p}\right)=\sum\left(u+k y_{3}\right)^{p}=0
\end{gathered}
$$

implies $\operatorname{Cor}_{\left\langle a_{3}, c\right\rangle}^{G_{1}^{1}}\left(u^{p}\right)=0$ (in fact, there is no essential element of degree $2 p$ in $\left.H^{*}\left(B G_{1}^{1} ; \mathbf{Z} / p\right)\right)$. Moreover $\operatorname{Cor}_{\left\langle a_{3}, c\right\rangle}^{G_{1}^{1}}(u)=f=0$. Hence we know

$$
\operatorname{Cor}_{\tilde{A}}^{B}\left(u^{p}\right)=0 \text { and } \operatorname{Cor}_{\tilde{A}}^{B}(u)=0 .
$$

Remark 4.1. For the group $G_{1}^{2}$, we note that

$$
\left\{w(2)^{\prime} x_{1} x_{2}\right\} \doteq\left(\left\{p u^{p(p-1)}\right\}\{p u\}\right) \doteq r\left(\left\{p u^{p(p-1)}\right\}\right) f \doteq\left\{f^{2} u^{p(p-1)-2}\right\} f .
$$

There contains errors in Theorem 8.18 in [T-Y3]. The elements $z_{p(p-1)-1} z$ and $\left\{w(2)^{\prime} x_{1} x_{1}\right\}$ in $H^{*}\left(B G_{1} ; \mathbf{Z} / p\right)$ should be deleted. Ignoring the assumption $|b| \neq$ 
$2 p(p-1)+2$ in Lemma 8.18 occurred the errors. Hence $\eta=0$ in Prop. 6 in [Mi], while the main theorem in $[\mathrm{Mi}]$ is of course correct.

Remark 4.2. Considering the restriction to $G_{\infty}^{1}$ and using the arguments in Lemma 7.3 below, we can prove

$$
\left\{f u^{p}\right\} \mid\left(G_{1}^{1} \times \mathbf{Z} / p\right) \doteq \operatorname{tr}(2) y_{3}^{p-1} .
$$

Now we study $m(i)$ for $n \leq i \leq p-1 \bmod (p)$.

Lemma 4.6. Let $n \leq i \leq p-1 \bmod (p)$. Then for the group $G_{\infty}^{n}$, the number $m(i)=n$, that is, $p^{n} u^{i}$ generates $I E_{\infty}^{0, *}$.

Proof. By induction, we assume the above fact for $n$. Consider the map of extensions

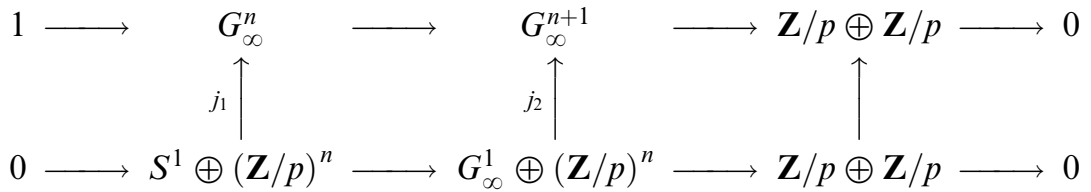

and the induced spectral sequences

$$
\begin{gathered}
E(n+1)_{2}^{*, *}=H^{*}\left(B(\mathbf{Z} / p \oplus \mathbf{Z} / p) ; H^{*}\left(B G_{\infty}^{n}\right)\right) \Rightarrow H^{*}\left(B G_{\infty}^{n+1}\right) \\
E\left(1^{\prime}\right)_{2}^{*, *}=H^{*}\left(\mathbf{Z} / p \oplus \mathbf{Z} / p ; H^{*}\left(B\left(S^{1} \oplus(\mathbf{Z} / p)^{n}\right)\right) \Rightarrow H^{*}\left(B\left(G_{\infty}^{1} \oplus(\mathbf{Z} / p)^{n}\right)\right) .\right.
\end{gathered}
$$

The differential of the transferred element is

$$
\begin{aligned}
d_{2}(\operatorname{tr}(i)) & =d_{2}\left(j_{1 !}\left(u^{i}\right)\right)=j_{2 !} d_{2}\left(u^{i}\right) \\
& =j_{2 !}\left(i u^{i-1} \otimes z_{12}(1)\right)=i j_{1 !}\left(u^{i-1}\right) \otimes z_{12}(1)=i \operatorname{tr}(i-1) \otimes z_{12}(1)
\end{aligned}
$$

where $j$ i is the transfer map induced from an injection $j$.

We assume that $n+1 \leq i \leq p-1 \bmod (p)$, and so $n \leq i-1 \bmod (p)$. This means that $\operatorname{tr}(i-1)$ generates $I E_{\infty}^{0,2(i-1)}$ and $\operatorname{tr}(i-1) \neq 0$ by inductive assumption. Thus $\operatorname{tr}(i)$ is not a permanent cycle in $E(n+1)_{r}^{* * *}$. Hence $p^{n+1} u^{i}$ generates $I E_{\infty}^{0,2 i}$ for $H^{*}\left(B G_{\infty}^{n+1}\right)$.

COROLlary 4.7 ([T-Y2] Theorem 5.2). $\quad p^{m+n} \mid \exp \left(H^{*}\left(B G_{m}^{n}\right)\right)$.

Proof. Note that $I E_{\infty}^{0, *}$ is generated by $p^{n} u^{p^{n}-1}$ (resp. $\left.u^{p^{n}}\right)$ when $*=$ $2\left(p^{n}-1\right)\left(\right.$ resp. $\left.*=2 p^{n}\right)$. Therefore the differential in (3.2) is

$$
d_{2}\left(\left(p^{n} u^{p^{n}-1}\right) \otimes z\right)=p^{n} u^{p^{n}-1} p^{m} u=p^{m+n} u^{p^{n}} .
$$

\section{Morava $K$-theory}

In this section, we compute the Morava $K$-theory of the group $\tilde{G}$. Let us write the infinitive term $E_{\infty}^{*, 0}$ by $A$, i.e., 


$$
A=S_{4} \otimes \Lambda_{4} /\left(w(1), w(2), z(1), z(2), z(3), w(2)^{\prime} \beta\left(x_{1} x_{2}\right)\right) .
$$

Write by $A_{i}, 0 \leq i \leq 4$, the $S_{4}$-submodule of $A$ generated by $i$-th product $x_{j_{1}} \cdots x_{j_{i}}$ of odd degree generators. In particular, $A_{0}=S_{4} /(w(1), w(2))$.

We consider the additive decomposition

$$
A_{0}=B_{0} \oplus C_{0} \quad \text { with } B_{0}=A_{0} /\left(w_{12}(1)\right), C_{0}=A_{0}\left\langle w_{12}(1)\right\rangle
$$

where $A_{0}\langle w\rangle$ means the $A_{0}$-submodule of $A$ generated by $w$ (while $A_{0}\{w\}$ means the free $A_{0}$-module). Here we have

$$
B_{0} \cong S_{12} /\left(w_{12}(1)\right) \otimes S_{34} /\left(w_{34}(1)\right), \quad C_{0} \cong S_{4} /\left(w(1), w(2)^{\prime}\right)\left\{w_{12}(1)\right\}
$$

where $S_{i j}=\mathbf{Z} / p\left[y_{i}, y_{j}\right], w_{i j}(1)=y_{i}^{p} y_{j}-y_{i} y_{j}^{p}$, so that $w(1)=w_{12}(1)+w_{34}(1)$ and $w(2)=w(2)^{\prime} w_{12}(1)$.

We also consider the decomposition of $A_{+}$such that

$$
\begin{gathered}
A_{+}=B_{+} \oplus C_{+} \quad \text { with } B_{+}=A_{+} /\left(z_{12}(1), z_{12}(2), x_{1} x_{2}, x_{3} x_{4}\right), \\
C_{+}=A\left\langle z_{12}(1), z_{12}(2), x_{1} x_{2}, x_{3} x_{4}\right\rangle
\end{gathered}
$$

Let $B=B_{0} \oplus B_{+}$and $C=C_{0} \oplus C_{+}$so that $A=B \oplus C$. Let us write

$$
\begin{gathered}
B_{i j}=B_{i j, 0} \oplus B_{i j,+} \text { where } B_{i j, 0}=S_{i j} /\left(w_{i j}(1)\right), \\
B_{i j,+}=S_{i j}\left\{x_{i}, x_{j}\right\} /\left(z_{i j}(1), z_{i j}(2), x_{i} x_{j}\right) \\
\cong S_{i j}\left\{x_{i}, x_{j}\right\} /\left(y_{i} x_{j}-y_{j} x_{i}, y_{i}^{p} x_{j}-y_{j} x_{i}^{p}, x_{i} x_{j}\right) \cong S_{i j} /\left(y_{j i}\right)\left\{x_{i}\right\} \oplus \mathbf{Z} / p\left[y_{j}\right]\left\{x_{j}\right\}
\end{gathered}
$$

so that $B \cong B_{12} \otimes B_{34}$. Here

$$
y_{j i}=w_{j i}(1) / y_{i}=y_{j}^{p}-y_{i}^{p-1} y_{j} .
$$

The $Q_{k}$-action is given by $Q_{k} x_{i}=y_{i}^{p^{k}}$. Hence $Q_{k}: B_{i j,+} \rightarrow B_{i j, 0}=S_{i j} /\left(w_{i j}(1)\right)$ is injective since $w_{i j}(1)=y_{i} y_{j i}$. Then we can easily compute the $Q_{k}$ homology

$$
H\left(B_{i j}, Q_{k}\right) \cong S_{i j} /\left(y_{i}^{p^{k}}, y_{j}^{p^{k}}, w_{i j}(1)\right) \text {. }
$$

By Kunneth formula, we have;

LEMMA 5.1.

$$
H\left(B ; Q_{k}\right) \cong S_{4} /\left(y_{1}^{p^{k}}, \ldots, y_{4}^{p^{k}}, w_{12}(1), w_{34}(1)\right) .
$$

Next we will study $H\left(C ; Q_{k}\right)$. Recall

$$
C_{0}=S_{4} /\left(w(1), w(2)^{\prime}\right)\left\{w_{12}(1)\right\} \quad C_{+}=\left(S_{4} \otimes \Lambda_{4}\right)\left\langle z_{12}(1), z_{12}(2), x_{1} x_{2}, x_{3} x_{4}\right\rangle .
$$

For ease of notation, let us write $D=S_{4} /\left(w(1), w(2)^{\prime}\right)$. We already know $z_{12}(1)$ generates the $D$-module in $C_{+}$since $\beta\left(x_{1} x_{2}\right)=z_{12}(1)$.

LEMMA 5.2. $w(2)^{\prime} z_{12}(2)=0$.

Proof. In $S_{12}$, we have $P^{1} w_{12}(1)=0$ and 


$$
P^{1} w_{12}(2)=P^{1}\left(y_{1}^{p^{2}} y_{2}-y_{1} y_{2}^{p^{2}}\right)=y_{1}^{p^{2}} y_{2}^{p}-y_{1}^{p} y_{2}^{p^{2}}=w_{12}(1)^{p} .
$$

Since $w_{12}(2)=w_{12}(2)^{\prime} w_{12}(1)$, we get $P^{1} w_{12}(2)^{\prime}=w_{12}(1)^{p-1}$. Hence in $C$, we get

$$
0=P^{1}\left(w(2)^{\prime} z_{12}(1)\right)=\left(w_{12}(1)^{p-1}-w_{34}(1)^{p-1}\right) z_{12}(1)+w(2)^{\prime} P^{1} z_{12}(1)
$$

The first term of the righthand side of the above equation is zero since $w_{12}(1)=$ $-w_{34}(1)$ in $A$. The fact $P^{1} z_{12}(1)=z_{12}(2)$ implies the result $w(2)^{\prime} z_{12}(2)=0$.

Thus we get the map $Q_{1}: D\left\langle z_{12}(1)\right\rangle \rightarrow D\left\{w_{12}(1)\right\}$. Here $Q_{1}\left(z_{12}(1)\right)=w_{12}(1)$ and its image is a $D$-free module. Therefore this map is an isomorphism, i.e., $z_{12}(1)$ generates a free $D$-module. Since $Q_{0}\left(z_{12}(2)\right)=w_{12}(1), z_{12}(2)$ also generates a free $D$-module. Moreover $Q_{0}\left(z_{12}(1)\right)=0$ and $Q_{1}\left(z_{12}(2)\right)=0$. This means that $D\left\langle z_{12}(1)\right\rangle$ and $D\left\langle z_{12}(2)\right\rangle$ have no intersection except for zero. Thus we have

\section{Lemma 5.3. $\quad C_{1} \cong D\left\{z_{12}(1)\right\} \oplus D\left\{z_{12}(2)\right\}$.}

Next consider the module $C_{2}$, Note that $x_{1} z_{12}(1)=-y_{2} x_{1} x_{2}$ and $x_{3} z_{12}(1)=$ $-x_{3} z_{34}(1)=y_{4} x_{3} x_{4}$. Similar fact holds for $z_{12}(2)$. Thus we get

$$
C_{2}=S_{4}\left\langle x_{1} x_{2}, x_{3} x_{4}\right\rangle \cong S_{4}\left\langle x_{1} x_{2}, f\right\rangle .
$$

We have the map $Q_{0}: D\left\langle x_{1} x_{2}\right\rangle \rightarrow D\left\{z_{12}(1)\right\}$ with $Q_{0}\left(x_{1} x_{2}\right)=z_{12}(1)$. While $w(2)^{\prime} x_{1} x_{2} \neq 0$, but the fact $y_{1} x_{1} x_{2}=x_{1} z_{12}(1)\left(y_{3} x_{1} x_{2}=-y_{3} x_{3} x_{4}=-x_{3} z_{34}(1)\right)$ implies that $S_{4}^{+}\left\langle x_{1} x_{2}\right\rangle$ is a $D$-module. Hence we have the isomorphism

LEMMA 5.4. There is an additive isomorphism

$$
C_{2} \cong D\left\{x_{1} x_{2}\right\} \oplus \mathbf{Z} / p\left\{w(2)^{\prime} x_{1} x_{2}\right\} \oplus S_{4} /\left(w_{i j}(1) \mid i<j\right)\{f\} .
$$

Proof. We already know the module $S_{4}\langle f\rangle$ from Theorem 2.5. The kernel of the map $Q_{0}: C_{2} \rightarrow D\left\{z_{12}(1)\right\}$ is direct sum of $\mathbf{Z} / p\left\{w(2)^{\prime} x_{1} x_{2}\right\}$ and the $S_{4}$-module generated by $f$.

The generators $x_{i_{1}} x_{i_{2}} x_{i_{3}} \in C_{3}$ are represented as $x_{i} f$, e.g., $x_{1} x_{2} x_{3}=f x_{3}$. The $S_{4}$-submodule generated by $x_{i} f, 1 \leq i \leq 4$ is still given in Theorem 2.5

$$
C_{3} \cong H(E, z(2))^{\text {odd }} \cong S_{4}\left\{x_{i} f \mid 1 \leq i \leq 4\right\} /\left(y_{j i} x_{i} f \mid i \neq j\right) .
$$

We also note that $Q_{0}: C_{3} \rightarrow S_{4}^{+} /\left(w_{i j}(1) \mid i<j\right)\{f\}$ is an isomorphism. The fact

$$
C_{4} \cong \mathbf{Z} / p\left\{x_{1} x_{2} x_{3} x_{4}=f^{2}\right\}
$$

is also given in Theorem 2.5.

First note that $Q_{k}\left(f^{2}\right)=0$, since this element is represented as the transfer.

LEMMA 5.5. $H\left(S_{4} /\left(w_{i j}(1)\right)\{f\} \oplus C_{3} ; Q_{k}\right) \cong S_{4} /\left(w_{i j}(1), y_{i}^{p^{k}}\right)\{f\}$. 
Proof. Exchanging $y_{i} y_{j}^{p}$ by $y_{i}^{p} y_{j}$ if $i>j$, each element $a \in S_{4} /\left(w_{i j}(1)=\right.$ $\left.y_{i}^{p} y_{j}-y_{i} y_{j}^{p}\right)$ is uniquely represented as

$$
a=\sum a_{I} y_{I} \quad \text { with } a_{I} \in \mathbf{Z} / p, y_{I}=y_{m}^{i_{m}} \cdots y_{4}^{i_{4}}, m<\cdots<4
$$

such that $i_{m} \neq 0$ and $0 \leq i_{j}<p$ for all $m<j$.

Similarly using the relation $0=y_{j i} x_{i}=\left(y_{j}^{p}-y_{i}^{p-1} y_{j}\right) x_{i}$, each element $b \in C_{3}$ is uniquely written as

$$
b=\sum b_{I} z_{I} f \quad \text { with } b_{I} \in \mathbf{Z} / p, z_{I}=x_{m} y_{m}^{i_{m}} \cdots y_{4}^{i_{4}}, m<\cdots<4
$$

such that $0 \leq i_{j}<p$ for all $m<j$. The $Q_{k}$ action is given by

$$
Q_{k}(b)=\sum b_{I} y_{m}^{p^{k}+i_{m}} y_{m+1}^{i_{m+1}} \cdots y_{4}^{i_{4}} f .
$$

Hence if $b \neq 0$ in $C_{3}$, then $Q_{k}(b) \neq 0$ also in $S_{4} /\left(w_{i j}(1)\right)\{f\}$. This proves the lemma.

Lemma 5.6. Let $k$ be an algebraic closed field of $\operatorname{ch}(k)=p$. For each $\lambda \in k$, the sequence $\left(w(1), w(2)^{\prime}, y_{3}-\lambda y_{4}, y_{4}\right)$ is regular in $S_{4} \otimes k$.

Proof. The sequence is regular if and only if the dimension of the variety

$$
\operatorname{dim}_{k} \operatorname{Var}\left(w(1), w(2)^{\prime}, y_{3}-\lambda y_{4}, y_{4}\right)=4-4=0 .
$$

Letting $y_{3}=y_{4}=0$, we only need to show $\operatorname{dim}_{k} \operatorname{Var}\left(w_{12}(1), w_{12}(2)^{\prime}\right)=0$ where $w_{12}(2)^{\prime}=\left(y_{1}^{p^{2}} y_{2}-y_{1} y_{2}^{p^{2}}\right) /\left(y_{1}^{p} y_{2}-y_{1} y_{2}^{p}\right)$. The regularity of $\left(w_{12}(1), w_{12}(2)^{\prime}\right)$ in $S_{2}$ is well known, in fact, these elements are Dickson invariants

$$
\mathbf{Z} / p\left[y_{1}, y_{2}\right]^{S L_{2}(Z / p)}=\mathbf{Z} / p\left[w_{12}(1), w_{12}(2)^{\prime}\right] .
$$

Let us write $w_{12}(k)=\left(y_{1}^{p^{k}} y_{2}-y_{1} y_{2}^{p^{k}}\right)=Q_{k} z_{12}(1)=Q_{k} Q_{0}\left(x_{1} x_{2}\right)$. Then

Lemma 5.7. Suppose that $a w_{12}(k)+b w_{12}(k-1)^{p}=0$ in $S_{4} /(w(1), w(2))$.

$$
a=\left(w_{12}(k-1)^{p} / w_{12}(1)\right) c, \quad b=\left(w_{12}(k) / w_{12}(1)\right) c \quad \text { for } c \in S_{4} /\left(w(1), w(2)^{\prime}\right) .
$$

Proof. When $k \leq 2$, the theorem is almost immediate. We assume $k \geq 3$. Suppose that $a w_{12}(k) / w_{12}(1)+b w_{12}(k-1)^{p} /\left(w_{12}(1)\right)=0$ in $D=$ $S_{4} /\left(w(1), w(2)^{\prime}\right)$. We have the decomposition

$$
w_{12}(k) / w_{12}(1)=\prod_{\lambda \in F_{p} k-F_{p}}\left(y_{2}-\lambda y_{1}\right) .
$$

Let $y_{2}-\lambda y_{1}=0$ for $\lambda \in F_{p^{k}}-F_{p}$. Then by the supposition we get 


$$
0=b w_{12}(k-1)^{p} / w_{12}(1)=b \lambda^{\prime} y_{1}^{p^{k}-p}
$$

in $\quad S_{4} \otimes \bar{F}_{p} /\left(w(1), w(2)^{\prime}, y_{2}-\lambda y_{1}\right) \quad$ and $\quad \lambda^{\prime} \neq 0 \in \bar{F}_{p} \quad$ because $\quad F_{p^{k}}-F_{p} \quad$ and $F_{p^{k-1}}-F_{p}$ have no intersection in $\bar{F}_{p}$. Since $\left(w(1), w(2)^{\prime}, y_{2}-\lambda y_{1}, y_{1}\right)$ is regular, we have $b=0$ in $S_{4} \otimes \bar{F}_{p} /\left(w(1), w(2)^{\prime}, y_{2}-\lambda y_{1}\right)$ and we can take $b=$ $\left(y_{2}-\lambda y_{1}\right) c^{\prime} \in S_{4} \otimes \bar{F}_{p} /\left(w(1), w(2)^{\prime}\right)$. Continuing this argument for all other $\lambda \in F_{p^{k}}-F_{p}$ and we get $b=w_{12}(k) / w_{12}(1) c$.

Apply the similar arguments for $y_{2}-\mu y_{1}, \mu \in F_{p^{k-1}}$, we get the lemma.

Lemma 5.8. The homology $H\left(C_{0} \oplus C_{1} \oplus D\left\{x_{1} x_{2}\right\} ; Q_{k}\right)$ is isomorphic to

$$
D /\left(w_{12}(k) / w_{12}(1), w_{12}(k-1)^{p} / w_{12}(1)\right)\left\{w_{12}(1)\right\} .
$$

Proof. We will show that the following sequence is exact

$$
0 \rightarrow D\left\{x_{1} x_{2}\right\} \stackrel{Q_{k}}{\rightarrow} D\left\{z_{12}(1), z_{12}(2)\right\} \stackrel{Q_{k}}{\rightarrow} D\left\{w_{12}(1)\right\} .
$$

The $Q_{k}$-operations are given

$$
\begin{gathered}
Q_{k}\left(z_{12}(1)\right)=Q_{k}\left(y_{1} x_{2}-y_{2} x_{1}\right)=y_{1} y_{2}^{p^{k}}-y_{2} y_{1}^{p^{k}}=-w_{12}(k) \\
Q_{k}\left(z_{12}(2)\right)=Q_{k}\left(y_{1}^{p} x_{2}-y_{2}^{p} x_{1}\right)=\left(y_{1}^{p} y_{2}^{p^{k}}-y_{1}^{p^{k}} y_{2}^{p}\right)=-w_{12}(k-1)^{p} .
\end{gathered}
$$

Hence if $c_{1}=a z_{12}(1)+b z_{12}(2) \in C_{1}$ is in the kernel $\operatorname{Ker}\left(Q_{k}\right)$, then from Lemma 5.7, we have

$$
c_{1}=c\left(w_{12}(k-1)^{p} /\left(w_{12}(1)\right) z_{12}(1)+w_{12}(k) /\left(w_{12}(1)\right) z_{12}(2)\right) \text { with } c \in D
$$

which is just $c Q_{k}\left(x_{1} x_{2}\right)$, indeed,

$$
Q_{0} Q_{k}\left(x_{1} x_{2}\right)=w_{12}(k) \text { and } Q_{1} Q_{k}\left(x_{1} x_{2}\right)=w_{12}(k-1)^{p}
$$

imply that

$$
Q_{k}\left(x_{1} x_{2}\right)=w_{12}(k-1)^{p} /\left(w_{12}(1)\right) z_{12}(1)+w_{12}(k) /\left(w_{12}(1)\right) z_{12}(2)
$$

since $Q_{1} z_{12}(1)=w_{12}(1), Q_{0} z_{12}(2)=w_{12}(1)$, and $Q_{0} z_{12}(1)=0, Q_{1} z_{12}(2)=0$.

Since $w_{12}(k)$ and $w_{12}(k-1)^{p}$ are in $\operatorname{Ideal}\left(y_{i}^{p^{k}}\right)$ in $A_{0}$ we have

Corollary 5.9. $H\left(B \oplus C_{0} \oplus C_{1} \oplus D\left\{x_{1} x_{2}\right\}, Q_{k}\right) \cong S_{4} /\left(w(1), w(2), y_{i}^{p^{k}}\right)$.

COROLlary 5.10. $H\left(E_{\infty}^{*, 0}, Q_{k}\right)$ is generated as an $S_{4}$-module by $1, w(2)^{\prime} x_{1} x_{2}$, $f$ and $f^{2}$.

Recall the isomorphism $E_{\infty}^{*, p s} \cong C_{3} \oplus S_{4} /\left(w_{i j}(1)\{f\} \oplus \mathbf{Z} / p\left\{f^{2}\right\}\right.$. Hence its cohomology is still given in Lemma 5.5. As for elements $\left\{f^{2} u^{s}\right\}$, we may assume that its $Q_{k}$-action is trivial because $H\left(E_{\infty}^{*, 0} ; Q_{k}\right)$ is generated by even dimensional elements. Thus we get 
THEOREM 5.11. There is an isomorphism

$$
\begin{aligned}
& H\left(H^{*}\left(B G_{\infty}^{2} ; \mathbf{Z} / p\right) ; Q_{k}\right) \cong \mathbf{Z} / p\left[u^{p^{2}}\right] \otimes\left(S_{4} /\left(w(1), w(2), y_{i}^{p^{k}}\right) \oplus \mathbf{Z} / p\left\{w(2)^{\prime} x_{1} x_{2}\right\}\right. \\
&\left.\oplus \underset{s}{\oplus} \mathbf{Z} / p\left\{f^{2} u^{s}\right\} \oplus \bigoplus_{t} S_{4} /\left(w_{i j}(1), y_{i}^{p^{k}}\right)\left\{f u^{p t}\right\}\right)
\end{aligned}
$$

where $0 \leq s \neq(p-1) \bmod p$ and $s \neq p^{2}-2$ and $0 \leq t \leq p-2$. Thus this homology is generated by even dimensional elements. Hence we have

$$
K(k)^{*}\left(B G_{\infty}^{2}\right) \cong K(k)^{*} \otimes H\left(H^{*}\left(B G_{\infty}^{2} ; \mathbf{Z} / p\right), Q_{k}\right) .
$$

Next consider the cases of finite groups $G_{m}^{2}, m \geq 2$. By arguments after (3.2), we see

$$
H\left(H^{*}\left(B G_{m}^{2} ; \mathbf{Z} / p\right), Q_{k}\right) \cong H\left(H^{*}\left(B G_{\infty}^{2} ; \mathbf{Z} / p\right), Q_{k}\right) \otimes \Lambda(z) .
$$

We consider the Atiyah-Hirzebruch spectral sequence

$$
E_{2}^{* * *}=H^{*}\left(B G_{m}^{2} ; K(k)^{*}\right) \Rightarrow K(k)^{*}\left(B G_{m}^{2}\right) .
$$

Recall $K(k)^{*} \cong \mathbf{Z} / p\left[v_{k}, v_{k}^{-1}\right]$. Since the first nonzero differential is the form $d_{2 p^{k}-1}(x)=v_{k} \otimes Q_{k}(x)$, we still have the $E_{2 p^{k}}^{* *}$-term. Since all elements in $H\left(H^{*}\left(B G_{\infty}^{2} ; \mathbf{Z} / p\right), Q_{k}\right)$ are permanent cycles in the above spectral sequence, we only need to study $d_{r} z$.

Consider the injection $\mathbf{Z} / p^{m} \subset G_{m}^{2}$. The Morava $K$-theory is

$$
K(k)^{*}\left(B \mathbf{Z} / p^{m}\right) \cong K(k)^{*}[u] /\left(\left[p^{m}\right](u)\right) .
$$

Here $[p](u)=v_{k} u^{p^{k}}$ implies $\left[p^{m}\right](u)=v_{k}^{1+p^{k}+\cdots+p^{(m-1) k}} u^{p^{m k}}$. Thus in the AtiyahHirzebruch spectral sequence converging $K(k)^{*}(B \mathbf{Z} / p)$, the differential

$$
d_{2 p^{m k}-1}(z)=v_{k}^{1+p^{k}+\cdots+p^{(m-1) k}} u^{p^{m k}} .
$$

Thus we get

THEOREM 5.12. Let $m \geq 2$. Then

$$
K(k)^{*}\left(B G_{m}^{2}\right) \cong K(k)^{*}\left(B G_{\infty}^{2}\right) /\left(u^{p^{m k}}\right) .
$$

\section{BP-theory}

Let $B P^{*}(-)$ be the Brown-Peterson cohomology theory with the coefficient ring $B P^{*}=\mathbf{Z} / p\left[v_{1}, \ldots\right],\left|v_{i}\right|=-2\left(p^{n}-1\right)$. Since $K(k)^{\text {odd }}\left(B G_{m}^{2}\right)=0$ for $m \geq 2$, we also have $B P^{\text {odd }}\left(B G_{m}^{2}\right)=0$ from the theorem by Ravenel-Wilson-Yagita [R-W-Y]. In this section we will study $B P^{*}\left(B G_{m}^{2}\right)$ more explicitly.

Recall that $\tilde{E}_{r}^{*, *}$ (resp. $I E_{r}^{*, *}$ ) is the Hochschild-Serre spectral sequence converging to $H^{*}\left(B G_{\infty}^{2} ; \mathbf{Z} / p\right)$ (resp. $\left.H^{*}\left(B G_{\infty}^{2}\right)\right)$. From Lemma 3.13, we already know that $I E_{\infty}^{+, 0} \cong \beta E_{\infty}^{+, 0} \oplus \mathbf{Z} / p\left\{f, f^{2}, w(2)^{\prime} x_{1} x_{2}\right\}$. The decomposition $\tilde{E}_{\infty}^{+, 0} \cong$ $B^{+} \oplus C$ is given in $\S 5$ with 


$$
H\left(B^{+}, \beta\right) \cong 0 \text { and } H(C ; \beta) \cong \mathbf{Z} / p\left\{w(2)^{\prime} x_{1} x_{2}, f, f^{2}\right\} .
$$

Note that $B$ and $C$ are closed under the Bockstein operation. The Bockstein images of $C$ is

$$
\beta C \cong D\left\{w_{12}(1), z_{12}(1)\right\} \oplus\left(S_{4}^{+} /\left(w_{i j}(1)\{f\}\right) .\right.
$$

Here $Q_{1} z_{12}(1)=w_{12}(1)$. The Bockstein of $B$ is

$$
S_{4} /\left(w_{12}(1), w_{34}(1)\right) \oplus S_{4}\left\langle\beta\left\{x_{s} x_{t} \mid 1 \leq s \leq 2,3 \leq t \leq 4\right\}\right\rangle .
$$

Lemma 6.1. If $0 \neq x \in B_{2}$ in the notation in $\S 5$, then $Q_{1} Q_{0} x \neq 0$ in $B_{0}$.

Proof. Each element $x$ in $B_{2}$ is expressed as (recall the arguments before Lemma 5.1)

$$
x=a_{13} x_{1} x_{3}+a_{14} x_{1} x_{4}+a_{23} x_{2} x_{3}+a_{24} x_{2} x_{4}
$$

where $a_{13} \in S_{4} /\left(y_{21}, y_{43}\right), \quad a_{23} \in S_{34} /\left(y_{43}\right) \otimes \mathbf{Z} / p\left[y_{2}\right], \quad a_{14} \in S_{12} /\left(y_{12}\right) \otimes \mathbf{Z} / p\left[y_{4}\right]$, $a_{24} \in \mathbf{Z} / p\left[y_{2}, y_{4}\right]$.

Suppose that $Q_{1} Q_{0} x=0$ in $B_{0}=S_{4} /\left(w_{12}(1), w_{34}(1)\right)$. First let $y_{1}=y_{3}=0$. Then $Q_{1} Q_{0} x=Q_{1} Q_{0} a_{24} x_{2} x_{4}=a_{24} w_{24}(1)$. But $w_{24}(1)=y_{2}^{p} y_{4}-y_{2} y_{4}^{p}$ is a nonzero divisor in $\mathbf{Z} / p\left[y_{2}, y_{4}\right]$. Hence $a_{24}=0$.

Next let $y_{1}=0$. Then $Q_{1} Q_{0} x=a_{23} w_{23}(1)$. But $y_{2}-\lambda y_{3}$ is a nonzero divisor in $S_{34} /\left(y_{34}\right) \otimes \mathbf{Z} / p\left[y_{2}\right]$ because the dimension of the variety

$$
\operatorname{Var}\left(y_{43}, y_{2}-\lambda y_{3}\right)=\bigcup_{\mu}\left(y_{4}-\mu y_{3}, y_{2}-\lambda y_{3}\right)
$$

is just one. Hence $a_{23}=0$. Similarly letting $y_{3}=0$, we have $a_{14}=0$.

Lastly, consider $Q_{1} Q_{0} x_{1} x_{3}$. The dimension of the variety is

$$
\operatorname{Var}\left(y_{21}, y_{34}, y_{1}-\lambda y_{3}\right)=\bigcup_{\mu, \mu^{\prime}} \operatorname{Var}\left(y_{2}-\mu y_{1}, y_{4}-\mu^{\prime} y_{3}, y_{1}-\lambda y_{3}\right)
$$

is also just one. Hence $w_{13}(1)=y_{1}^{p} y_{3}-y_{1} y_{3}^{p}$ is also nonzero divisor in $S_{4} /\left(y_{21}, y_{43}\right)$. So $a_{13}=0$.

Since $Q_{1} Q_{0}\left(x_{s} x_{t}\right)=w_{s t}(1)$ and $Q_{1}\left(z_{12}(1)\right)=w_{12}(1)$, we have;

COROLlary 6.2. $Q_{1}\left(I E_{\infty}^{+, 0}\right) \cong S_{4}\left\langle w_{i j}(1) \mid i<j\right\rangle$.

We also known $I E_{\infty}^{+, 2 p} \cong S_{4} /\left(w_{i j}(1)\right)\{f\}$. Considering the AtiyahHirzebruch spectral sequence

$$
E_{2}^{*, *}=H^{*}\left(B G_{\infty}^{2} ; B P^{*}\right) \Rightarrow B P^{*}\left(B G_{\infty}^{2}\right) .
$$

The first nonzero differential is $d_{2 p-1}(x)=v_{1} \otimes Q_{1}(x)$. The term $E_{2 p}^{*, *}$ is generated by even dimensional elements. Hence we have;

THEOREM 6.3. The graded ring $\operatorname{gr} B P^{*}\left(B G_{\infty}^{2}\right)$ is isomorphic to 
$\left(B P^{*} \otimes S_{4} /\left(w(1), w(2), v_{1} w_{i j}(1)\right) \oplus B P^{*} \otimes(F \oplus U)\right) \otimes \mathbf{Z}\left[\left\{u^{p^{2}}\right\}\right]$ where $F=S_{4}^{+} /\left(w_{i j}(1)\right)\left\{f u^{s p} \mid 1 \leq s \leq p-2\right\}, U=\mathbf{Z}\left\{u_{t} \mid 0 \leq t \leq p^{2}-1\right\}$,

$$
u_{t}=\left\{\begin{array}{l}
\{1\} \quad t=0 \\
\left\{p u^{t}\right\} \quad t=0,1 \bmod (p) \text { and } 0<t \neq p(p-1)+1 \\
\left\{p^{2} u^{t}\right\} \quad 2 \leq t \leq p-1 \bmod (p) \text { or } t=p(p-1)+1 .
\end{array}\right.
$$

Corollary 6.4. All $B P^{*}$-linear relations in $B P^{*}\left(B G_{\infty}^{2}\right)$ are deduced from the relations in $B P^{*}(B V)$.

Proof. Since $[p]\left(y_{i}\right)=p y_{i}+v_{1} y_{i}^{p}+\cdots=0$ in $B P^{*}(B V)$, we have the relation in $B P^{*}(B V)$,

$$
y_{j}[p]\left(y_{i}\right)-y_{i}[p]\left(y_{j}\right)=v_{1}\left(y_{i}^{p} y_{j}-y_{i} y_{j}^{p}\right)+\cdots=v_{1} w_{i j}(1)+\cdots=0 .
$$

We consider the cases of finite groups $G_{m}^{2}, m \geq 2$. Recall that

$$
H^{*}\left(B G_{m}^{2}\right) \cong I E_{\infty}^{+, 0} \otimes \Lambda(z) \oplus I E_{\infty}^{0, *} /\left(\left\{p^{m} u\right\}\right) .
$$

We easily see that $I E_{\infty}^{0, *} /\left(\left\{p^{m} u\right\}\right)$ is generated by $u_{t}, 0 \leq t \leq p^{2}-1$ and $\left\{u^{p^{2}}\right\}$ with

$$
\exp \left(u_{t}\right)= \begin{cases}p^{m-1} & (t=2 \bmod (p) \text { but } t \neq p(p-1)+2) \\ & \text { or }(t=1) \text { or }(t=p(p-1)+1) \\ p^{m} & (3 \leq t \leq p-1 \bmod (p)) \text { or }(1 \bmod (p) \text { but } \\ & t \neq 1, \neq p(p-1)+1) \text { or }(t=p(p-1)+2) \\ p^{m+1} & (t=p s, 0<s<p) \\ p^{m+2} & \left(t=p^{2}\right) .\end{cases}
$$

THEOREM 6.5. For $m \geq 2$, we have the isomorphism

$$
\operatorname{gr} B P^{*}\left(B G_{m}^{2}\right) \cong \operatorname{gr} B P^{*}\left(B G_{\infty}^{2}\right) /\left(v_{1}^{s_{1}} y_{i} u^{p^{m}}, v_{2}^{s_{2}} w_{i j}(1) u^{p^{2 m}}, \exp \left(u_{t}\right) u_{t}\right)
$$

where $s_{k}=1+p^{k}+\cdots+p^{(m-1) k}$.

Proof. We consider the Atiyah-Hirzebruch spectral sequence

$$
E_{2}^{*, *}=H^{*}\left(B G_{m}^{2} ; B P^{*}\right) \Rightarrow B P^{*}\left(B G_{m}^{2}\right) \text {. }
$$

The first nonzero differential is $d_{2 p-1}(x)=v_{1} \otimes Q_{1}(x)$. The $2 p$-term is

$$
\begin{aligned}
& E_{2 p}^{*, *} \cong\left(B P^{*} \otimes S_{4} /\left(w(1), w(2), v_{1} w_{i j}(1)\right) \oplus B P^{*} \otimes(F)\right) \otimes \Lambda(z) \\
& \oplus B P^{*} \otimes U /\left(\exp \left(u_{t}\right) u_{t}\right) \otimes \tilde{\mathbf{Z}} /\left(p^{m+2}\right)\left[u^{p^{2}}\right]
\end{aligned}
$$

where $\tilde{\mathbf{Z}} /\left(p^{m+2}\right)\left[u^{p^{2}}\right]$ means $\mathbf{Z}\left[u^{p^{2}}\right] /\left(p^{m+2} u^{p^{2}}\right)$. By $K(1)^{*}(-)$ theory, the next nonzero differential is $d_{2 p^{m}-1}\left(y_{i} z\right)=v_{1}^{s_{1}} y_{i} u^{p^{m}}$. The last nonzero differential is $d_{2 p^{2 m}-1}\left(w_{i j}(1) z\right)=v_{2}^{s_{2}} w_{i j}(1) u^{p^{2 m}}$ from $K(2)^{*}(-)$ theory. Thus we get the theorem. 
7. $B P^{*}\left(B p_{+}^{1+4}\right)$

In this section, we will study the $B P$-theory of the case $m=1$, i.e., $G_{1}^{2}=p_{+}^{1+4}$. The integral cohomology is (the integral version of Lemma 2.9)

$$
\operatorname{gr} H^{*}\left(B G_{1}^{2}\right) \cong\left(\left(\operatorname{Ker}(f) \mid H^{*}\left(B G_{\infty}^{2}\right)\right)\{z\} \oplus H^{*}\left(B G_{\infty}^{2}\right) /(f) .\right.
$$

Recall (see $\$ 6$ also)

$$
\left\{\begin{array}{l}
I E_{\infty}^{+, 0} /(f) \cong \beta B \oplus D\left\{w_{12}(1), z_{12}(1)\right\} \\
\operatorname{Ker}(f) \mid I E_{\infty}^{+, 0} \cong S_{4}\left\langle w_{s t}(1), \beta\left(x_{s} x_{t}\right) \mid 1 \leq s \leq 2<t \leq 4\right\rangle \oplus \beta C \\
I E_{\infty}^{+, 2 p s} /(f) \cong \operatorname{Ker}(f) \mid I E_{\infty}^{+, 2 p s} \cong S_{4}^{+} /\left(w_{i j}(1)\left\{f u^{p s}\right\}\right) \quad 1 \leq s \leq p-2 .
\end{array}\right.
$$

Hence from Lemma 6.1 and the arguments before the lemma, we have

$$
\left\{\begin{array}{l}
H\left(I E_{\infty}^{+, 0} /(f), Q_{1}\right) \cong S_{4}^{+} /\left(w_{i j}(1)\right) \\
H\left(\operatorname{Ker}(f) \mid I E_{\infty}^{+, 0}, Q_{1}\right) \cong S_{4}^{+} /\left(w_{i j}(1)\right)\{f z\} \\
H\left(I E_{\infty}^{+, 2 p s} \otimes \Lambda(z), Q_{1}\right) \cong S_{4}^{+} /\left(w_{i j}(1)\left\{f u^{p s}\right\} \Lambda(z)\right.
\end{array}\right.
$$

Thus we can prove that

Lemma 7.1. The homology $H\left(\operatorname{gr} H^{*}\left(B G_{1}^{2}\right), Q_{1}\right)$ is isomorphic to $\left(\left(S_{4}^{+} /\left(w_{i j}(1)\right) \otimes\left(\Lambda(f z) \oplus \mathbf{Z} / p\left\{u^{p} f, \ldots, u^{p(p-2)} f\right\} \otimes \Lambda(z)\right) \oplus U\right) \otimes \tilde{\mathbf{Z}} / p^{3}\left[u^{p^{2}}\right]\right.$.

We will study the Atiyah-Hirzebruch spectral sequence

$$
E_{2}^{* * *}=H^{*}\left(\operatorname{gr} H^{*}\left(B G_{1}^{2}\right) ; \tilde{K}(1)^{*}\right) \Rightarrow \tilde{K}(1)^{*}\left(B G_{1}^{2}\right)
$$

where $\tilde{K}(1)^{*}(-)$ is the integral $K$-theory with the coefficient ring $\tilde{K}(1)^{*}=$ $\mathbf{Z}_{(p)}\left[v_{1}, v_{1}^{-1}\right]$. The first nonzero differential is also

$$
d_{2 p-1}(x)=v_{1} \otimes Q_{1}(x)
$$

but $Q_{1}(x)$ is considered as an element in $\operatorname{gr} H^{*}\left(B G_{1}^{2}\right)$. We want to prove the following lemma;

LEMMA 7.2. $d_{2 p-1}\left(y_{i} f z u^{p(s-1)}\right) \doteq v_{1} y_{i} f u^{p s} \quad$ for $1 \leq s \leq p-2$ and hence $Q_{1}\left(y_{i} f z u^{p(s-1)}\right) \doteq y_{i} f u^{p s}$ in $H^{*}\left(B G_{1}^{2} ; \mathbf{Z} / p\right)$.

To prove this lemma, we prepare some lemmas. For a compact group $G$, it is known that $\tilde{K}(1)^{\text {odd }}(B G)=0$ and $\tilde{K}(1)^{*}(B G)$ is torsion free by the Atiyah theorem. Hence $K(1)^{\text {odd }}(B G)=0$. Moreover it is given

$$
\operatorname{dim}_{K(1)^{*}} K(1)^{*}\left(B G_{1}^{2}\right)=p^{4}+p-1
$$

by Brunetti [B1]. In [B2], he also showed that the Euler characteristic for $K(n)^{*}$ theory has the property $\chi_{n, p}\left(G_{2}^{2}\right)=p^{n} \chi_{n, p}\left(G_{1}^{2}\right)$. Indeed, from Theorem 5.11 and Theorem 5.12, we know $\operatorname{dim}_{K(1)^{*}} K(1)^{*}\left(B G_{2}^{2}\right)=p^{5}+p^{2}-p$. 
Given $\lambda_{i} \in F_{p}^{\times}, 1 \leq i \leq 4$ with $\lambda_{1} \lambda_{2}=\lambda_{3} \lambda_{4}$, let $g=g\left(\lambda_{1}, \ldots, \lambda_{4}\right)$ be the automorphism of $G_{1}^{2}$ defined by

$$
a_{i} \mapsto a_{i}^{\lambda_{i}}, \quad c \mapsto c^{\lambda_{1} \lambda_{2}} .
$$

Then the induced map $g^{*}$ defines the automorphism of $H^{*}\left(B G_{1}^{2}\right)$, and moreover the automorphism of the Hochschild-Serre spectral sequence converging to $H^{*}\left(B G_{1}^{2}\right)$ so that

$$
y_{i} \mapsto \lambda_{i} y_{i} \quad u \mapsto \lambda_{1} \lambda_{2} u .
$$

Indeed this gives the (weight) decomposition of the spectral sequence.

For a sequence $I=\left(i_{1}, \ldots, i_{4}\right)$, let $y^{I}=y_{1}^{i_{1}} \cdots y_{4}^{i_{4}}$. Suppose that in $\tilde{K}(1)^{*}\left(B G_{1}^{2}\right)$, there is a relation

$$
p y^{I}\left\{u^{p t}\right\}=v_{1}^{s} \sum_{K} a_{K} y^{K} \bmod \left(p^{2}, v_{1}^{s+1}\right),
$$

where $y^{K} \neq 0 \in S_{4} /\left(y_{i}^{p}\right), 0 \neq a_{K} \in \mathbf{Z} / p$. Let $J=K-I$. Applying $g^{*}$ on the above equation, we have

$$
\left(\lambda_{1} \lambda_{2}\right)^{t}=\lambda_{1}^{j_{1}} \lambda_{2}^{j_{2}} \lambda_{3}^{j_{3}}\left(\lambda_{1} \lambda_{2} / \lambda_{3}\right)^{j_{4}}
$$

Hence we get

$$
j_{1}=j_{2}, \quad j_{3}=j_{4}, \quad t=j_{1}+j_{3} \bmod (p-1) .
$$

On the other hand, by dimensional reason,

$$
2 t=\left|u^{p t}\right|=\left|v_{1}^{s}\right|+\left|y^{J}\right|=4 i_{1}+4\left(t-i_{1}\right) \bmod (2(p-1)) .
$$

This means $t=0 \bmod (p-1)$. Similar facts hold for the differentials since the action $g^{*}$ is compatible with the differentials of the spectral sequence. Thus we get

Lemma 7.3. If $(*)$ holds or $d_{r}\left(y^{I} z f u^{(t-2) p}\right)=$ righthandside of $(*)$, then $t=0 \bmod (p-1)$ and letting $J=K-I$,

$$
j_{1}=j_{2}=p-1-j_{3}=p-1-j_{4} \bmod (p-1) .
$$

Lemma 7.4. In $(*)$, letting $t=0$, we have $s \geq 2$.

Proof. If $s=1$, then by [Y1], there is an element $x \in H^{*}\left(B G_{1}^{2} ; \mathbf{Z} / p\right)$ such that $Q_{0}(x)=y^{I}$ and $Q_{1} x=y^{K}$. But $Q_{1} x_{i}=y_{i}^{p} \in S_{4} \otimes \Lambda_{4}$ so this contradicts to $y^{K} \neq 0$ in $S_{4} /\left(y_{i}^{p}\right)$.

Let us write by $I V$ the vector space in $S_{4} /\left(y_{i}^{p}\right)$ $I V=\left\{y \in S_{4} /\left(y_{i}^{p}\right) \mid \operatorname{deg}(y)>4(p-1)\right\} \oplus \mathbf{Z} / p\left\{\left(y_{1} y_{2}\right)^{j}\left(y_{3} y_{4}\right)^{p-1-j} \mid 0 \leq j \leq p-1\right\}$.

LEMma 7.5. $\operatorname{dim}_{\mathbf{Z} / p}\left(S_{4} /\left(y_{i}^{p}, I V\right)\right)>\left(p^{4}+p-1\right) / 2$. 
Proof. First note that $\operatorname{dim}_{\mathbf{Z} / p}\left(S_{4} /\left(y_{i}^{p}\right)\right)=p^{4}$. Since the largest degree of $S_{4} /\left(y_{i}^{p}\right)$ is $8(p-1)$, by the duality the $t$-dimensional homogeneous parts are

$$
\operatorname{dim}_{\mathbf{Z} / p}\left(S_{4} /\left(y_{i}^{p}\right)\right)^{t}=\operatorname{dim}_{\mathbf{Z} / p}\left(S_{4} /\left(y_{i}^{p}\right)\right)^{8(p-1)-t} .
$$

The degree of $\left(y_{1} y_{2}\right)^{j}\left(y_{3} y_{4}\right)^{p-1-j}$ is of course $4(p-1)$ and it generates a $p$ dimensional $\mathbf{Z} / p$-vector space. Note $\operatorname{deg}(I V) \geq 4(p-1)$. The $4(p-1)$ homogeneous parts of $S_{4} /\left(y_{i}^{p}\right)$ is quite large, e.g., $\operatorname{dim}_{\mathbf{Z} / p}\left(S_{4} /\left(y_{i}^{p}\right)\right)^{4(p-1)}>p^{2}$. Since

$$
\begin{aligned}
\operatorname{dim}_{\mathbf{Z} / p}\{y \mid \operatorname{deg}(y) \leq 4(p-1)\} \\
=1 / 2 \operatorname{dim}_{\mathbf{Z} / p}\left\{S_{4} /\left(y_{i}^{p}\right)\right\}+1 / 2 \operatorname{dim}_{\mathbf{Z} / p}\{y \mid \operatorname{deg}(y)=4(p-1)\},
\end{aligned}
$$

we know

$$
\operatorname{dim}_{\mathbf{Z} / p}\left(S_{4} /\left(y_{i}^{p}, I V\right)\right)>p^{4} / 2+p^{2} / 2-p>\left(p^{4}+p-1\right) / 2 .
$$

Lemma 7.6. As $K(1)^{*}$-modules, we have the injection

$$
K(1)^{*} \otimes S_{4} /\left(y_{i}^{p}, I V\right) \subset K(1)^{*}\left(B G_{1}^{2}\right) .
$$

Proof. First we note that additively $\tilde{K}(1)^{*} \otimes S_{4} /\left(y^{p}, I V\right) \subset \tilde{K}(1)^{*}\left(B G_{1}^{2}\right)$, because all targets of differentials are in $I V$ by dimensional reasons and Lemma 7.3. If $0 \neq y \in S_{4} /\left(y_{i}^{p}, I V\right)$ is zero in $K(1)^{*}\left(B G_{1}^{2}\right)$, then there is $y^{\prime} \in \tilde{K}(1)^{*}\left(B G_{1}^{2}\right)$ such that

$$
p y^{\prime}=v_{1}^{s} y \quad \text { for } s \leq 2 .
$$

But this does not happen from Lemma 7.3 and the definition of $I V$.

Lemma 7.7. If $d_{2 p-1}\left(y_{i} z f\right)=0$, then $d_{4 p-3}\left(y_{i} z f\right)=0$ in the spectral sequence (7.2).

Proof. From Lemma 7.1, we can write

$$
d_{4 p-3}\left(y_{1} f z\right)=v_{1}^{2} \sum b_{J} y^{J} y_{1} u^{p} f \bmod \left(v_{1}^{3}\right) .
$$

If $|J| \geq 0$ and if there is $j_{i} \neq 0 \bmod (p-1)$, then from Lemma 7.3, we see $\left|y^{J}\right| \geq 4(p-1)$, and this contradicts to the dimensional reason. Hence all $j_{i}=0 \bmod (p-1)$ if $j_{1} \geq 0$. If $j_{1}=-1$, there is the case $y^{J} y_{1}=y_{2}^{p-2} y_{3} y_{4}$ by the similar arguments. Let us write

(**) $\quad d_{4 p-3}\left(y_{1} f z\right)=v_{1}^{2}\left(\left(\sum_{i} b_{i} y_{i}^{p-1}\right) y_{1}+b^{\prime} y_{2}^{p-2} y_{3} y_{4}\right) f u^{p} \bmod \left(v_{1}^{3}\right)$.

We consider the (twisted) automorphism $t w$ defined by

$$
t w: a_{1} \leftrightarrow a_{3}, \quad a_{2} \leftrightarrow a_{4}, \quad c \mapsto c,
$$


which induces

$$
t w^{*}: y_{1} \leftrightarrow y_{3}, \quad y_{2} \leftrightarrow y_{4}, \quad u \mapsto u
$$

on the spectral sequence. Applying $t w^{*}$ on $(* *)$, we get

$$
\begin{aligned}
& d_{4 p-3}\left(y_{3} f z\right) \\
& \quad=v_{1}^{2}\left(\left(b_{1} y_{3}^{p-1}+b_{2} y_{4}^{p-1}+b_{3} y_{1}^{p-1}+b_{4} y_{2}^{p-1}\right) y_{3}+b^{\prime} y_{4}^{p-2} y_{1} y_{2}\right) f u^{p} \bmod \left(v_{1}^{3}\right) .
\end{aligned}
$$

Since $y_{3} d_{4 p-3}\left(y_{1} f z\right)=y_{1} d_{4 p-3}\left(y_{3} f z\right)$, we know $b_{4}=b_{2}$ and $b^{\prime}=0$. We also have the other twisted map, e.g., $t w^{\prime}: a_{1} \leftrightarrow a_{4}$. Similarly, we get $b_{1}=b_{2}=b_{3}=b_{4}$.

We consider the other automorphism $f_{\lambda}$ of $G_{1}^{2}$ defined by

$$
f_{\lambda}: a_{3} \mapsto a_{3} a_{4}^{\lambda}, \quad f_{\lambda}: a \mapsto a \text { for } a=a_{i}, i \neq 3 \text { or } c
$$

which induces

$$
f_{\lambda}^{*}: y_{4} \mapsto y_{4}+\lambda y_{3}, \quad f_{\lambda}^{*}: y \mapsto y \quad \text { for } y=y_{i}, i \neq 4 \text { or } u .
$$

Apply $f_{\lambda}^{*}$ on $(* *)$ with $b_{i}=b$. Then the left hand side of $\left(f_{\lambda}^{*}-\mathrm{id}.\right)(* *)$ is zero, but the righthand side is

$$
v_{1}^{2} b\left(\left(y_{4}+y_{3}\right)^{p-1}-y_{4}^{p-1}\right) y_{1} f u^{p} \neq 0, \quad \text { if } b \neq 0 .
$$

Hence $b$ must be zero.

Proof of Lemma 7.2. If $d_{2 p-1}\left(y_{i} z f\right) \neq 0$, then it is $\lambda v_{1} y_{i} f u^{p}$ for $\lambda \neq 0 \in \mathbf{Z} / p$ by the dimensional reason. Suppose $d_{2 p-1}\left(y_{i} z f\right)=0$. Then from above lemma, $d_{4 p-3}\left(y_{i} f z\right)=0$. This means that all nonzero element in $\tilde{K}(1)^{*} \otimes S_{4} /\left(y_{i}^{p}, I V\right)$ are not targets of differentials. By arguments similar to the proof of Lemma 7.6 and Lemma 7.4, we can show

$$
K(1)^{*} \otimes S_{4} /\left(y_{i}^{p}, I V\right)\left\{1, f u^{p}\right\} \subset K(1)^{*}\left(B G_{1}^{2}\right) .
$$

The dimension of the left hand side $K(1)^{*}$-vector space is larger than $p^{4}+p-1$ by Lemma 7.5. This contradicts to the result of $\operatorname{dim}_{K(1)^{*}} K(1)^{*}\left(B G_{1}^{2}\right)$ by Brunetti. Thus we get $d_{2 p-1}\left(y_{i} z f\right) \doteq\left\{y_{i} u^{p} f\right\}$. By the induction on $s$ we get the lemma. q.e.d.

Therefore we get

$$
E_{2 p}^{*, *} \cong \tilde{K}(1)^{*} \otimes\left(S_{4}^{+} /\left(w_{i j}(1)\left\{1, u^{p(p-2)} z f\right\} \oplus U\right) \otimes \mathbf{Z} / p^{3}\left[u^{p^{2}}\right] .\right.
$$

From Theorem 5.12, we know $u^{p^{2}}=0 \in K(1)^{*}\left(B G_{2}^{2}\right)$. Hence so in $K(1)^{*}\left(B G_{1}^{2}\right)$. However from Lemma 7.3, there is no $y^{\prime} \in \tilde{K}(1)^{*}\left(B G_{1}^{2}\right)$ such that $p y^{\prime}=v_{1}^{s} y_{i} u^{p^{2}}$ since $y^{\prime} \in S_{4}^{+} /\left(w_{i j}(1)\right)$ or $y^{\prime} \in U$. (Note that there is such $y^{\prime} \in U$ for $v_{1}^{s} u^{p^{2}}$.) Hence for some $s$, the element $v_{1}^{s} y_{i} u^{p^{2}}$ is a target of differential in the spectral sequence. By dimensional reason we have

$$
d_{4 p-3}\left(y_{i} u^{p(p-2)} f z\right) \doteq v_{1}^{2} y_{i} u^{p^{2}} .
$$

Thus we get; 
COHOMOLOGY OF EXTRASPECIAL $p$-GROUPS FOR ODD PRIMES

LEMMA 7.8 .

$$
\begin{aligned}
& \operatorname{gr} \tilde{K}(1)^{*}\left(B G_{1}^{2}\right) \cong \tilde{K}(1)^{*} \otimes\left(S_{4}^{+} /\left(w_{i j}(1)\right) \oplus U \otimes \tilde{\mathbf{Z}} / p^{3}\left[u^{p^{2}}\right]\right), \\
& \operatorname{gr} K(1)^{*}\left(B G_{1}^{2}\right) \cong K(1)^{*} \otimes\left(S_{4} /\left(y_{i}^{p}\right) \oplus \mathbf{Z} / p\left\{u_{3}, \ldots, u_{p+1}\right\}\right) .
\end{aligned}
$$

Proof. We study elements in $U$. In $H^{*}\left(B G_{\infty}^{2}\right)$, we have

$$
u_{1}=\{p u\}=f, \quad u_{2}=\left\{p^{2} u^{2}\right\}=f^{2},
$$

which are zero in $H^{*}\left(B G_{1}^{2}\right)$ from (7.1). Relations $p^{3} u^{i}=v_{1} p^{2} u^{p+i-1}$ in $\tilde{K}(1)^{*}(B\langle c\rangle)$ give that for $U$ in $\tilde{K}(1)^{*}\left(B G_{1}^{2}\right)$, e.g., $p u_{3}=v_{1} u_{p+2}, p u_{4}=v_{1} u_{p+3}, \ldots$

Note that $\operatorname{dim}_{K(1)^{*}} K(1)^{*}\left(B G_{1}^{2}\right)$ is in fact $p^{4}+p-1$.

THEOREM 7.9. The $B P^{*}$-algebra gr $B P^{*}\left(B G_{1}^{2}\right)$ is isomorphic to the quotient of the free $B P^{*}$-algebra

$$
B P^{*} \otimes\left(S_{4}^{+} /(w(1), w(2)) \oplus U \oplus S_{4}^{+} /\left(w_{i j}(1)\right)\left\{f u^{p}, \ldots, f u^{p(p-2)}\right\}\right) \otimes \tilde{\mathbf{Z}} / p^{3}\left[u_{p^{2}}\right]
$$

by the following relations

$$
\left(v_{1} w_{i j}(1), v_{1} y_{i} f u^{s p}, v_{1}^{2} y_{i} u_{p^{2}}, v_{2} w_{i j}(1) u_{p^{2}}\right)
$$

Proof. We consider the Atiyah-Hirzebruch spectral sequence

$$
E_{2}^{*, *}=H^{*}\left(B G_{1}^{2} ; B P^{*}\right) \Rightarrow B P^{*}\left(B G_{1}^{2}\right) .
$$

The first nonzero differential is $d_{2 p-1}(x)=v_{1} \otimes Q_{1}(x)$, which was still given in the arguments for $\tilde{K}(1)^{*}$-theory.

$$
\begin{aligned}
E_{2 p}^{*, *} \cong & B P^{*} \otimes\left(U \oplus S_{4}^{+} /\left(w(1), w(2), v_{1} w_{i j}(1)\right) \oplus S_{4}\left\langle w_{i j}(1)\right\rangle /\left(v_{1}\right)\{z\}\right. \\
& \oplus S_{4}^{+} /\left(w(1), v_{1}\right)\left\{f u^{p}, \ldots, f u^{p(p-2)}\right\} \oplus S_{4}^{+} /\left(w_{i j}(1)\left\{f z u^{p(p-2)}\right\}\right) \otimes \tilde{\mathbf{Z}} / p^{3}\left[u^{p^{2}}\right] .
\end{aligned}
$$

Here note that $B P^{*} /\left(v_{1}\right) \otimes S_{4}\left\langle w_{i j}(1)\right\rangle\{z\}$ remains, while it disappears for $\tilde{K}(1)^{*}$ theory.

The next nonzero differential is $d_{4 p-3}\left(y_{i} u^{p(p-2)} f z\right) \doteq v_{1}^{2} y_{i} u^{p^{2}}$ same as the $\tilde{K}(1)^{*}$-theory. The last nonzero differential is

$$
d_{2 p^{2}-1} w_{i j}(1) z \doteq v_{2} w_{i j}(1) u^{p^{2}}
$$

which is given from $K(2)^{*}$-theory and ( $v_{2}$-version of ) Lemma 7.3.

Proof of Remark 4.2. First we consider the element $\left\{f u^{p}\right\}$ in $H^{*}\left(B G_{\infty}^{2} ; \mathbf{Z} / p\right)$. Recall that $[\mathrm{L}]$

$$
H^{\text {even }}\left(B G_{\infty}^{1}\right) / p \cong\left(S_{2} /\left(w_{12}(1)\right) \oplus \mathbf{Z} / p\{\operatorname{tr}(1), \ldots, \operatorname{tr}(p-1)\}\right) \otimes \mathbf{Z} / p\left[u_{p}\right]
$$

where $\operatorname{tr}(i)=\operatorname{Cor}_{\left\langle a_{1}, c\right\rangle}^{G_{\infty}^{1}}\left(u^{i}\right)$ and $u_{p}=\left\{u^{p}\right\}$. Since 


$$
\operatorname{Im} \rho\left(B P^{*}\left(B G_{\infty}^{1} \times B \mathbf{Z} / p\right)\right) \cong H^{\text {even }}\left(B G_{\infty}^{1}\right) \otimes \mathbf{Z} / p\left[y_{3}\right]
$$

for the Thom map $\rho: B P \rightarrow H \mathbf{Z}_{(p)}$, we can write

$$
\left\{f u^{p}\right\} \mid G_{\infty}^{1} \times \mathbf{Z} / p=\sum a\left(i^{\prime}, i^{\prime \prime}, J\right) \operatorname{tr}\left(i^{\prime}\right) u_{p}^{i^{\prime \prime}} y^{J} .
$$

By arguments similar to the proof of Lemma 7.3, we have

$$
i^{\prime}+i^{\prime \prime}+j_{1}=2, \quad j_{1}=j_{2}, \quad j_{3}=0 \bmod (p-1) .
$$

Hence by the dimensional reason, we can write

$$
(*)=\operatorname{tr}(1) u_{p}+\operatorname{tr}(2) a y_{3}^{p-1} .
$$

here we use the fact $y_{i} \operatorname{tr}(1)=y_{2} \operatorname{tr}(i)=0$ for $i<p-1$.

Now we consider the conjugation map $a_{4}^{*}$ on $H^{*}\left(B G_{\infty}^{2} ; \mathbf{Z} / p\right)$ (or $\left.H^{*}\left(B G_{\infty}^{1} \times \mathbf{Z} / p ; \mathbf{Z} / p\right), H^{*}\left(B\left(\left\langle a_{1}, a_{3}, c\right\rangle\right) ; \mathbf{Z} / p\right)\right)$ which induces

$$
a_{4}^{*}: u \mapsto u+y_{3}, \quad y_{i} \mapsto y_{i} .
$$

This action is invariant on the cohomology of $G_{\infty}^{2}$, and so is on $(*)$

$$
\left(a_{4}^{*}-1\right) \operatorname{tr}(1) u_{p}=\operatorname{Cor}\left(u+y_{3}\right) a_{4}^{*} u_{p}-\operatorname{Cor}(u) u_{p}=\operatorname{tr}(1)\left(a_{4}^{*}-1\right) u_{p} .
$$

We already know $u_{p} \mid\left\langle a_{1}, c\right\rangle=u^{p}-y_{1}^{p-1} u$. By the same argument as the proof of Proposition 4.5, we have

$$
\left(a_{4}^{*}-1\right) u_{p}=y_{3}^{p}-\chi y_{3} \quad \text { where } \chi=\operatorname{Cor}_{\left\langle a_{1}, c\right\rangle}^{G_{1}^{1}}\left(u^{p-1}\right)+y_{2}^{p-1} .
$$

On the other hand

$$
\left(a_{4}^{*}-1\right) \operatorname{tr}(2)=\operatorname{Cor}\left(\left(u+y_{3}\right)^{2}\right)-\operatorname{Cor}\left(u^{2}\right)=2 \operatorname{tr}(1) y_{3} .
$$

Hence we have

$$
\left(a_{4}^{*}-1\right)(*)=\operatorname{tr}(1)\left(y_{3}^{p}-\chi y_{3}\right)+2 a \operatorname{tr}(1) y_{3}^{p} .
$$

Here it is known that $\chi \operatorname{tr}(1)=0([\mathrm{~L}])$. Thus $a=-1 / 2$ and we get $(*)=$ $\operatorname{tr}(1) u_{p}-1 / 2 \operatorname{tr}(2) y_{3}^{p-1}$. Consider the restriction $(*) \mid G_{1}^{1} \times \mathbf{Z} / p$, and we have the remark since $\operatorname{tr}(1)=0$ in $H^{*}\left(B G_{1}^{1} ; \mathbf{Z} / p\right)$. q.e.d.

\section{Algebraic cobordism and Chow ring}

Let $X$ be a smooth algebraic variety over C. Recently Levine-Morel [L-M1,2] defined an algebraic cobordism $\Omega^{*}(X)$ having following properties.

(1) There is the natural map $\rho: \Omega^{*}(X) \rightarrow M U^{*}(X)$ such that $\Omega^{*}=$ $\Omega^{*}(p t) \cong M U^{*}(p t)$ where $M U^{*}(-)$ is the complex cobordism theory.

(2) $\Omega^{*}(X) \otimes_{\Omega^{*}} \mathbf{Z} \cong C H^{* / 2}(X)$; the classical Chow ring.

(3) $\Omega^{*}(X) \otimes_{\Omega^{*}} \tilde{K}(1)^{*} \cong K_{0}(X) \otimes \tilde{K}(1)^{*}$; where $K_{0}(X)$ is the Grothendieck group of algebraic bundle over the variety $X$. 
Let $G$ be an algebraic group over $\mathbf{C}$, Totaro [To1,2] defines the Chow ring $C H^{*}(B G)$ of the classifying space as a limit of algebraic varieties. He conjectured that

$$
C H^{* / 2}(B G)_{(p)} \cong B P^{*}(B G) \otimes_{B P^{*}} \mathbf{Z}_{(p)} .
$$

In particular he showed above conjecture for $* \leq 4$ ([To2] Corollary 3.5).

Recall that except for elements in $F=S_{4}^{+} /\left(w_{i j}(1)\right)\left\{f u^{p s}\right\}$ in Theorem 6.3, all elements in $B P^{*}\left(B G_{\infty}^{2}\right)$ are represented by transferred Chern classes, and hence come from the algebraic cobordism where transfers and Chern classes exist. Hence we only need to see whether $f u^{p s}$ are in the Chow ring or not.

THEOREM 8.1. $\left\{f u^{p}\right\} \in B P^{*}\left(B G_{\infty}^{2}\right)$ comes from the algebraic cobordism.

COROLlary 8.2. When $p=3$, the natural maps $\rho: \Omega^{*}\left(B G_{m}^{2}\right) \rightarrow B P^{*}\left(B G_{m}^{2}\right)$ are epic for all $m \geq 1$ or $m=\infty$.

Proof of Theorem 8.1. By Totaro (Theorem 3.1 in [To2]), $K_{0}(B G) \otimes$ $\tilde{K}(1)^{*} \cong \tilde{K}(1)^{*}(B G)$. From Theorem 6.3, $f u^{p s}$ is nonzero in $\tilde{K}(1)^{*}\left(B G_{\infty}^{2}\right)$. Hence from (3) there is $f_{s} \in \Omega^{*}\left(B G_{\infty}^{2}\right)$ with $\rho\left(f_{s}\right)=v_{1}^{t} f u^{p s}$. Now consider the case $s=1$. Note that $\Omega^{*}(X)$ is generated by positive degree elements as a $\Omega^{*}$ module from (2). Hence $t=0,1$. If $t=1$, then $\left|f_{s}\right|=4$ and this contradicts to Totaro's conjecture for $*=4$. Thus $t=0$ and we have the theorem. q.e.d.

\section{REFERENCES}

[B1] M. Brunetti, The $K(n)$-Euler characteristic of extraspecial $p$-groups. J. Pure and Appl. Algebra 155 (2001), 105-113.

[B2] M. BRUnetTI, Higher Euler characteristics for almost extraspecial p-groups. Contemporary Math. 293 (2002), 69-74.

[G] D. J. Green, Calculations related to the integral cohomology of extraspecial p-groups. preprint (1996).

[L] I. J. Leary, The integral cohomology rings of some $p$ groups. Math. Proc. Cambridge Philos. Soc. 110 (1991), 245-255.

[L-M1] M. Levine and F. Morel, Coborsime algébrique I. C. R. Acad. Sci. Paris 332 (2001), $723-728$.

[L-M2] M. Levine and F. Morel, Coborsime algébrique II. C. R. Acad. Sci. Paris 332 (2001), $815-820$.

[Mi] P. A. MiNH, Essential cohomology and extraspecial p-groups. Trans. AMS 353 (2000), 1937-1957.

[R-W-Y] D. C. Ravenel, W. S. Wilson AND N. Yagita, Brown-Peterson cohomology from Morava $K$-theory. $K$-theory 15 (1998), 147-199.

[Sc-Y] B. Schuster And N. Yagita, Morava $K$-theory of extraspecial 2-groups. Proc. AMS. 132 (2004), 1229-1239.

[T-Y1] M. TEZuka AND N. Yagita, The varieties of the mod $p$ cohomology rings of extra special p-groups for an odd prime $p$. Math. Proc. Cambridge Phil. Soc. 94 (1983), 449-459.

[T-Y2] M. Tezuka and N. Yagita, Cohomology of finite groups and the Brown-Peterson cohomology. Lecture Notes in Math. 1370 (1989), 396-408. 
[T-Y3] M. Tezuka AND N. Yagita, Calculations in $\bmod p$ cohomology of extra special $p$-groups I. Contemporary Math. 158 (1994), 281-306.

[To1] B. Totaro, Torsion algebraic cycles and complex cobordism. J. Amer. Math. Soc. 10 (1997), 467-493.

[To2] B. Totaro, The Chow ring of classifying spaces. Proc. of Symposia in Pure Math. "Algebraic $K$-theory" (1997: University of Washington, Seattle) 67 (1999), 248-281.

[Y1] N. YAGITA, On relations between Brown-Peterson cohomology and the ordinary mod $p$ cohomology theory. Kodai Math. J. 7 (1984), 273-285.

[Y2] N. YAGITA, Localization of the spectral sequence converging to the cohomology of an extra special $p$-group for odd prime $p$. Osaka J. Math. 35 (1998), 83-116.

Department of Mathematics

FACULTY OF EDUCATION

IBARAKI UNIVERSITY

Mito, IBARAKI, JAPAN

E-mail address: yagita@mx.ibaraki.ac.jp 